

\section{AVALIAÇÃO DA CAPACIDADE PREDITIVA DE MODELOS ARIMA E VAR-VEC: O CASO DA DEMANDA POR ENERGIA ELÉTRICA NO RIO GRANDE DO SUL}

\section{EVALUATION OF THE PREDICTIVE CAPACITY OF ARIMA AND VAR-VEC MODELS: THE CASE OF ELECTRICITY DEMAND IN RIO GRANDE DO SUL}

\author{
iD Gérson dos Santos Nunes ${ }^{1}$ \\ (D) Viviane Leite Dias de Mattos ${ }^{2}$ \\ (iD) Andréa Cristina Konrath ${ }^{3}$ \\ iD Luiz Ricardo Nakamura ${ }^{4}$ \\ iD Antônio Cezar Bornia ${ }^{5}$ \\ (iD) Paulo Siga Thomaz ${ }^{6}$
}

\section{Mestre}

Instituto Federal de Educação, Ciência e Tecnologia do Rio Grande do Sul - IFRS.

Rio Grande, Rio Grande do Sul - Brasil.

gerson.sn85@gmail.com

2 Doutora

Universidade Federal do Rio Grande - FURG.

Rio Grande, Rio Grande do Sul - Brasil.

viviane.leite.mattos@gmail.com

${ }^{3}$ Doutora

Universidade Federal de Santa Catarina - UFSC Florianópolis, Santa Catarina - Brasil. andreack@gmail.com

\section{Doutor}

Universidade Federal de Santa Catarina - UFSC Florianópolis, Santa Catarina - Brasil.

luiz.nakamura@ufsc.br

\section{${ }^{5}$ Doutor}

Universidade Federal de Santa Catarina - UFSC Florianópolis, Santa Catarina - Brasil.

cezar.bornia@ufsc.br

\section{${ }^{6}$ Mestre}

Universidade Federal do Rio Grande - FURG.

Rio Grande, Rio Grande do Sul - Brasil.

paulosigathomaz@gmail.com

Recebido em: 04 jun. 2020

Aprovado em: 13 jul. 2020
Resumo: O presente estudo apresenta a modelagem da demanda de energia elétrica no estado do Rio Grande do Sul para os três principais setores consumidores: residencial, comercial e industrial, utilizando o modelo vetorial autorregressivo, complementado pelo modelo vetorial de correção dos erros. Nesta modelagem, também foram utilizadas informações a respeito da tarifa de energia elétrica, PIB, preços de eletrodomésticos e preço de materiais e equipamentos elétricos. A capacidade preditiva dos modelos ajustados foi comparada com a do modelo obtido pela modelagem de Box-Jenkins, em especial, o modelo autorregressivo integrado de médias móveis (ARIMA). Para o ajuste dos modelos, foram utilizados dados do período 1971-2010, tendo sua validação realizada no período 2011-17. De maneira geral, para os três setores consumidores, a melhor capacidade preditiva foi obtida a partir dos modelos ARIMA. Entretanto, os outros modelos o suplantaram para a previsão um passo à frente.

Palavras-chave: Séries temporais. Modelo Box-Jenkins. Modelo de vetores autorregressivos. Modelo de correção de erros vetoriais.

Abstract: This paper presents the modelling of electricity demand in State of Rio Grande do Sul for the three main consumer sectors: residential, commercial and industrial, through the autoregressive vector model, complemented by the error correction vector model. In this approach, we also considered information regarding energy tariff, GDP, appliances and electrical material and equipment prices. The predictive capacity of all fitted models was compared to the Box-Jenkins framework, specifically, with the autoregressive integrated moving average (ARIMA) models. All models were fitted using data from 1971 to 2010, and their validation were performed from 2011 up to 2017. In general, for all three consumer sections, the best predictive capacity was returned by the ARIMA models. Nevertheless, the other models performed better on one-step-ahead predictions.

Keywords: Time series. Box-Jenkins model. Autoregressive vector model. Error correction vector model. 


\section{Introdução}

De acordo com a Agência Nacional de Energia Elétrica [ANEEL] (2008), a energia elétrica se apresenta como uma das mais versáteis e apropriadas fontes de energia, estando diretamente ligada aos índices de desenvolvimento socioeconômico de uma região. Alguns estudos, entre os quais Andrade e Lobão (1997), Schmidt e Lima (2004) e Silveira (2017), já mostraram que o crescimento do produto interno bruto (PIB) está diretamente relacionado com o crescimento da sua demanda, razão pela qual fica evidente que um país necessita investir em sua matriz energética para alcançar o seu desenvolvimento econômico.

Neste cenário, a modelagem da demanda de energia elétrica possibilita não apenas caracterizar o seu padrão de comportamento, mas fazer previsões, podendo fornecer subsídios para o planejamento da infraestrutura de uma região, de modo a não impedir ou retardar o seu desenvolvimento econômico. Vale salientar que o tempo esperado entre a constatação da necessidade de aumentar a produção de energia elétrica e a sua efetiva disponibilização para consumo fica entre três e cinco anos, o que enfatiza a necessidade de um bom sistema de planejamento (Ferreira, Braga \& Mendes, 2006).

Uma das alternativas frequentemente utilizada na literatura para a modelagem da demanda de energia elétrica é o modelo vetorial autorregressivo (VAR), um método multivariado que analisa a relação de longo prazo entre as variáveis consideradas na análise, complementado pelo modelo vetorial de correção dos erros (VEC), que ajusta o modelo encontrado para previsões de curto prazo, originando assim a abordagem VAR-VEC.

Embora Andrade e Lobão (1997), Schmidt e Lima (2004), Mattos e Lima (2005), Leite (2006), Irffi et al. (2009), Viana e Silva (2014) e Silveira (2017) tenham utilizado diferentes proxies e métodos de estimação dos parâmetros do modelo, assim como séries históricas de diferentes comprimento e memória para modelar a demanda de energia elétrica de diferentes regiões, o fizeram por meio do método VAR-VEC. Nestes estudos, os autores consideraram que esta demanda poderia ser estimada a partir de um modelo multiplicativo oriundo da função de Cobb-Douglas (Ferreira \& Silva, 2015).

Esta é uma modelagem relativamente simples, que normalmente retorna previsões satisfatórias, e em relação a outros modelos multivariados, como é o caso das equações estruturais, apresenta a vantagem de considerar todas as variáveis como endógenas, ou seja, variáveis de saída do modelo que podem ser explicadas por variáveis externas ao modelo ou exógenas, mas que influenciam o mesmo (Bueno, 2011; Gujarati \& Porter, 2011). Entretanto, para estes autores, dependendo da quantidade de variáveis envolvidas no problema e do número de defasagens consideradas no modelo, a quantidade de coeficientes a serem estimados pode ser elevada, indicando ser necessária uma amostra maior para obtenção de estimativas confiáveis e robustas. Soma-se o fato de que a ordem de 
integração das variáveis envolvidas, preferencialmente, deve ser a mesma, o que nem sempre é possível na prática (Bueno, 2011; Gujarati \& Porter, 2011).

De acordo com Castro et al. (2016), a inclusão de variáveis que se inter-relacionam com a variável de interesse, aumenta a capacidade de previsão. Entretanto, a exemplo do que acontece com todas as técnicas multivariadas, nestas análises existe a necessidade de utilização de outras séries históricas além da série da demanda de energia elétrica, o que, de certa forma, poderia aumentar o grau de incerteza contido nos dados, prejudicando a capacidade preditiva do modelo obtido.

Para Amaro et al. (2017), a previsão da demanda de energia de curto prazo é mais difícil de ser realizada porque envolve uma grande influência de vários fatores sociais e econômicos. Segundo González-Romera et al. (2008), nas previsões de médio e longo prazo essa influência fica diluída, resultando em picos e vales mais suaves na série analisada. Em vista disso, Amaro et al. (2017) sugerem que modelos univariados podem ser utilizados para prever a demanda de energia elétrica de maneira eficiente, como é o caso dos modelos propostos em Box-Jenkins (1976). No trabalho de Amaro et al. (2017), bons resultados referentes à série de demanda de energia elétrica no Brasil foram obtidos.

Assim, o presente estudo tem como objetivo apresentar a modelagem da demanda de energia elétrica no estado do Rio Grande do Sul para os três principais setores consumidores (residencial, comercial e industrial), utilizando o método multivariado VAR, complementado ou não pelo VEC, e o método univariado obtido pela modelagem de Box-Jenkins, mais precisamente, o modelo autorregressivo integrado de médias móveis (ARIMA).

\section{Os modelos}

\subsection{Metodologia VAR-VEC}

O modelo vetorial autoregressivo de ordem $p, \operatorname{VAR}(p)$, para $n$ variáveis é dado por

$$
X_{t}=\Phi_{0}+\Phi_{1} X_{t-1}+\cdots+\Phi_{p} X_{t-p}+a_{t}
$$

em que $X_{t}$ é um vetor de ordem $(n \times 1)$, composto pelo $t$-ésimo valor das variáveis analisadas, $\Phi_{0}$ é um vetor de ordem ( $\left.n \times 1\right)$, composto por constantes, $\Phi_{k}$ é uma matriz de ordem $(n \times n)$ composta por elementos $\varphi_{i j k}, i=1,2, . ., n, j=1,2, . ., n, k=1,2, . ., p$ e $a_{t}$ é um vetor de ordem $(n \times 1)$, composto pelos t-ésimos erros aleatórios.

Com o intuito de ajustar o modelo obtido pela Equação (1) para previsões de curto prazo, aplicase o modelo vetorial de correção de erros (VEC), onde o vetor de cointegração de longo prazo é usado como variável explicativa do termo de correção de erros. Matematicamente, subtraindo $X_{t-1}$ em ambos os lados da Equação (1), para $n$ variáveis, tem-se a Equação (2): 


$$
\Delta X_{t}=\pi_{0}+\pi X_{t-1}+\pi_{1} \Delta X_{t-1}+\cdots+\pi_{p} \Delta X_{t-p}+\epsilon_{t}
$$

em que: $\pi_{0}$ é um vetor de ordem $(n \times 1)$ composto por constantes, $\pi_{k}$ é uma matriz $(n \times n)$ de coeficientes, com elementos $\pi_{\mathrm{ijk},} i=1,2, . ., n, j=1,2, . ., n, k=1,2, . ., p, \pi$ é uma matriz com elementos $\pi_{\mathrm{jk}}$ tal que um ou mais elementos $\pi_{\mathrm{jk}} \neq 0$ e $\epsilon_{t}$ é um vetor $(n \times 1)$ composto pelos erros aleatórios $\varepsilon_{\mathrm{it}}$.

A aplicação conjunta dos dois modelos origina a abordagem VAR-VEC. Maiores detalhes podem ser encontrados em Bueno (2011).

\subsection{Metodologia de Box-Jenkins}

O modelo autorregressivo de médias móveis de ordens $p$ e $q$, ARMA $(p, q)$, não sazonal, ou seja sem um comportamento periódico, é dado pela Equação (3):

$$
X_{t}=c+\sum_{i=1}^{p} \emptyset_{i} X_{t-i}+\sum_{j=0}^{q} \theta_{j} \varepsilon_{t-j}
$$

em que: $X_{t}$ é o $t$-ésimo valor observado $(t=1,2, \ldots, T)(i=1,2, \ldots, p), c$ é uma constante, $i$ é a defasagem do processo auto-regressivo $(i=1,2, \ldots, p)$, $j$ é a defasagem do processo de médias móveis ( $=1,2, \ldots, q), \emptyset_{i}$ é o coeficiente da parcela auto-regressiva na i-ésima defasagem, $\vartheta_{j}$ é o coeficiente de médias móveis na j-ésima defasagem, $p$ é a ordem do processo autorregressivo, $q$ é a ordem do processo de médias móveis e $\varepsilon_{t}$ é o erro no t-ésimo tempo.

Uma generalização do modelo ARMA é o modelo autorregressivo integrado de médias móveis de ordens $p, d$ e $q$, ARIMA $(p, d, q)$, em que o valor $d$ adicional se refere ao grau de diferenciação.

\section{Materiais e métodos}

O estudo da demanda anual de energia elétrica no estado do Rio Grande do Sul foi realizado, separadamente, em cada um dos três setores consumidores: residencial (Dr), comercial (Dc) e industrial (Di), abrangendo o período de 1971 até 2017. A modelagem foi feita com os dados de 1971 até 2010, e foi utilizado o período de 2011 até 2017 para validação dos modelos encontrados. Além disso, considerou-se que toda a demanda de energia é efetivamente fornecida.

Os procedimentos descritos foram realizados com o auxílio dos softwares Gnu Regression, Econometrics and Times-Séries Library (Cottrell \& Lucchetti, 2018) e R (R Core Team, 2019), além da planilha Excel. Nas análises inferenciais, foram considerados os níveis de significância de 0,01e 0,05 e as estimações por intervalo foram realizadas com nível de confiança de 0,95. 


\subsection{Metodologia VAR-VEC}

As variáveis e respectivas proxies utilizadas neste artigo foram as mesmas utilizadas por Silveira (2017) e são apresentadas no Quadro 1, possibilitando que a previsão da demanda de energia elétrica seja feita a partir de valores defasados da própria demanda de energia elétrica, além de valores defasados de outras variáveis.

\section{Quadro 1}

Variáveis consideradas na construção do modelo VAR-VEC e respectivas fontes

\begin{tabular}{|c|c|c|}
\hline Variável & Descrição & Fonte \\
\hline Dr & Demanda total residencial & IPEA e EPE \\
\hline $\operatorname{Tr}$ & Tarifa média residencial & IPEA e ANEEL \\
\hline $\operatorname{Rr}$ & PIB-RS per capita & IBGE e FEE \\
\hline $\mathrm{Er}$ & $\begin{array}{c}\text { IPA-OG-eletrodomésticos e IPA- Origem - OG-DI - Produtos Industriais - } \\
\text { Indústria de Transformação - Máquinas e Equipamentos - Item } \\
\text { Eletrodomésticos }\end{array}$ & FGV e IPEA \\
\hline Dc & Demanda total comercial & IPEA e EPE \\
\hline Tc & Tarifa média comercial & IPEA e ANEEL \\
\hline $\mathrm{Rc}$ & PIB-RS de serviços - comércio & IBGE e FEE \\
\hline Ec & $\begin{array}{l}\text { IPA-OG Mat. Elétrico e IPA- Origem - OG-DI - Produtos Industriais - } \\
\text { Indústria de Transformação - Máquinas, Aparelhos e Materiais Elétricos }\end{array}$ & FGV e IPEA \\
\hline $\mathrm{Di}$ & Demanda total industrial & IPEA e EPE \\
\hline $\mathrm{Ti}$ & Tarifa média industrial & IPEA e ANEEL \\
\hline Ri & PIB-RS da indústria & IBGE e FEE \\
\hline $\mathrm{Ei}$ & $\begin{array}{c}\text { IPA-OG- Máquinas e Equipamentos Industriais e IPA- Origem - OG-DI - } \\
\text { Produtos Industriais - Indústria de Transformação - Máquinas e } \\
\text { Equipamentos }\end{array}$ & FGV e IPEA \\
\hline Si & $\begin{array}{c}\text { IPA-OG Combustível }{ }^{3} \text { e Lubrificantes. e IPA-EP - Bens Intermediários - } \\
\text { Combustíveis e Lubrificantes para a Produção }\end{array}$ & FGV e IPEA \\
\hline
\end{tabular}

Fonte: Elaborada com base em Silveira (2017).

O modelo multiplicativo, descrito pela Equação (4), foi adotado para explicar a demanda de energia elétrica, conforme Modiano (1984), Andrade e Lobão (1997), Schmidt e Lima (2004), Mattos e Lima (2005), Leite (2006), Lima (2011), Viana e Silva (2014) e Silveira (2017).

$$
D_{t}=k \cdot T_{t}^{\gamma} \cdot R_{t}^{\theta} \cdot E_{t}^{\alpha} \cdot S_{t}^{\beta}
$$

em que: $D_{t}$ é a demanda no $t$-ésimo período (residencial, comercial ou industrial), $k$ é uma constante, $T_{t}$ é a tarifa no t-ésimo período (residencial, comercial ou industrial), $R_{t}$ é a renda no $t$-ésimo período, $E_{t}$ é o preço dos eletrodomésticos no caso residencial ou o preço dos eletrointensivos nos casos comercial e industrial no $t$-ésimo período, $S_{t}$ é o preço de um bem substituto à energia elétrica no $t$ ésimo período, aplicado apenas no caso industrial, $\gamma$ é a elasticidade-preço em relação à tarifa, $\vartheta$ é a 
elasticidade-renda; $\alpha$ é a elasticidade-preço em relação aos eletrodomésticos, $\alpha$ é a elasticidade-preço em relação aos eletrodomésticos e $B$ é a elasticidade-preço em relação ao bem substituto à energia elétrica.

Também foi verificado o efeito de alguns contextos ambientais e econômicos que poderiam afetar a demanda de energia elétrica, considerados sob a forma de variáveis dummy, descritas no Quadro 2.

\section{Quadro 2}

Variáveis dummy consideradas na construção do modelo VAR-VEC

\begin{tabular}{|l|c|c|}
\hline \multicolumn{1}{|c|}{ Descrição da variável dummy } & Período & Valores \\
\hline Mudança na forma de determinação da tarifa & $1971-1992$ & 0 \\
\hline (DYr1, DYc1, DYi1) & $1993-2010$ & 1 \\
\hline Crise energética & $1971-2000$ & 0 \\
\hline (DYr2, DYc2, DYi2) & $2001-2002$ & 1 \\
\hline Período pré-planos econômicos - Cruzeiro & $2003-2010$ & 0 \\
\hline (DYr3, DYc3, DYi3) & $1971-1985$ & 1 \\
\hline Período plano econômicos - Troca de moeda & $1986-2010$ & 0 \\
\hline (DYr4, DYc4 e DYi4) & $1971-1985$ & 0 \\
\hline Período pós-planos econômicos - Real & $1986-1993$ & 1 \\
\hline (DYr5, DYc5, DYi5) & $1994-2010$ & 0 \\
\hline Crise econômica mundial & $1971-1993$ & 0 \\
\hline (DYr6, DYc6 e DYi6) & $1994-2010$ & 1 \\
\hline
\end{tabular}

Fonte: Elaborada pelos Autores.

Inicialmente os dados das variáveis independentes do modelo foram deflacionados por serem séries econômicas. Depois da alteração da métrica dos dados de todas as variáveis pela transformação logarítmica, com o objetivo de transformar o modelo multiplicativo em modelo aditivo, foi realizada uma análise exploratória com a construção do gráfico em linhas para analisar e comparar o comportamento das séries estudadas. Para traçá-lo, foi necessário adotar o ano de 1971 como ano de referência, atribuindo-Ihe o valor 100 para todas as variáveis. Os demais valores observados foram determinados em relação a este.

Após foram aplicados os testes ADF e KPSS para avaliar a estacionariedade. O teste de DickeyFulley Aumentado (ADF; Dickey \& Fulley, 1981) considera como hipótese nula a presença de raiz unitária, considerando que a série é não estacionaria, e o teste de Kwiatkowski-Phillips-Schmidt-Shin (KPSS; Kwiatkowski et al., 1992) considera como hipótese nula a ausência de raiz unitária, indicando a estacionaridade da série. Este procedimento foi executado de forma interativa, considerando o critério de Schwert (1989) para determinar o número máximo de defasagens a ser considerado. Na constatação 
de não estacionaridade foram realizadas diferenças sucessivas com repetição dos testes, até conseguila em cada uma das variáveis. Além disso, foram consideradas a inclusão de tendência e constante, apenas constante e nenhuma delas.

Na sequência ocorreu a especificação do modelo VAR com identificação do número de defasagens, a presença de termos determinísticos e a presença de variáveis dummy, identificando modelos candidatos. Nesta etapa foram utilizados os critérios de informação de Akaike, Schwarz, Hannan-Quinn. Seguindo, com a aplicação do modelo de Johansen (Johansen, 1988), são executados os testes do traço e os testes do máximo autovalor para verificar se existe vetor de cointegração entre as variáveis consideradas no modelo, sugerindo existência de relação de longo prazo entre elas. Deste forma, foi possível identificar a equação de cointegração, avaliando a significância estatística dos coeficientes. Em seguida, o modelo foi ajustado pelo método de correção de erros vetoriais (VEC), para descrever a relação de curto prazo entre as variáveis, permitindo fazer previsões.

Após ocorreu a avaliação das suposições do modelo teórico de: existência de autocorrelação nos resíduos por meio do teste de Ljung-Box (Ljung-Box, 1978), normalidade pelo teste de Jarque-Bera (Jarque \& Bera, 1987) e homogeneidade entre variâncias, mediante o teste de autoregressive conditional heteroskedasticity (ARCH) (Engle, 1982).

Por último, foi realizada a validação dos modelos VAR e VAR-VEC para previsão com a determinação dos indicadores: erro absoluto médio (MAE), erro quadrático médio (RMSE) e erro absoluto percentual médio (MAPE), além da estatística $U$ de Theil, com identificação dos melhores modelos para previsão. Neste momento foi desconsiderado o efeito da inflação.

Estas duas últimas etapas foram realizadas para resíduos e previsões obtidas apenas com aplicação do modelo VAR e para os resultados obtidos com a aplicação conjunta VAR-VEC,

O procedimento para a construção e seleção do modelo VAR-VEC, fundamentado em Bueno (2011), é descrito no fluxograma apresentado no Apêndice 1.

\subsection{Metodologia de Box-Jenkins}

Considerando a análise exploratória de dados (AED) realizada na etapa anterior para a variável demanda de energia elétrica, optou-se por continuar trabalhando com alteração de sua métrica pela aplicação da transformação logarítmica. Segue a construção do gráfico em linhas desta variável para uma primeira avaliação do comportamento dos dados em nível, avaliando a presença de tendência e estacionariedade. Foram também construídos os gráficos da Função de Autocorrelação (FAC) e da Função de Autocorrelação Parcial (FACP) para complementar a análise. Aplica-se o teste de Dickey-Fuller Aumentado (ADF), utilizando o mesmo ptotocolo descrito anteriormente para verificar a hipótese nula de que a série não é estacionária. Para complementá-lo, foi aplicado o teste KPSS para verificar a 
hipótese nula de que a série é estacionaria. Na constatação de não estacionaridade, foram realizadas diferenças sucessivas até consegui-la.

Após, são identificadas as ordens de modelos candidatos pela análise da FAC e da FACP da série identificada como estacionária. A confirmação dos modelos candidatos é feita por meio da função auto.arima.

Nestes modelos são determinados os coeficientes com avaliação de sua significância, além de ser definida uma hierarquia entre os mesmos por sua parcimonialidade, o que foi feito pelos critérios de informação de Akaike, de Schwarz e de Hannan-Quinn, os mesmos utilizados na modelagem VARVEC, o mesmo acontecendo com os procedimentos para a verificação das suposições do modelo teórico e para identificação dos melhores modelos para previsão.

Foram verificadas: existência de autocorrelação nos resíduos por meio do teste de Ljung-Box (Ljung-Box ,1978), normalidade pelo teste de Jarque-Bera (Jarque \& Bera, 1987) e homogeneidade entre variâncias pelo teste de autoregressive conditional heteroskedasticity (ARCH) (Engle, 1982).

Como indicadores de qualidade do modelo para previsão foram usados: erro absoluto médio (MAE), erro quadrático médio (RMSE) e erro absoluto percentual médio (MAPE), além da estatística U de Theil.

O procedimento para a construção e seleção do modelo ARIMA, fundamentado em Box et al (1994) e Gujarati e Porter (2011), é descrito no fluxograma apresentado no Apêndice 2.

\section{Resultados e discussões}

\subsection{Modelo VAR-VEC}

Após o deflacionamento das variáveis independentes e a aplicação da transformação logarítmica em todas as variáveis, foi elaborado o gráfico em linhas apresentado na Figura 1, onde é possível observar que para o setor residencial (Painel a), no período analisado, o preço dos eletrodomésticos diminuiu, o mesmo acontecendo com a tarifa, só que de maneira menos acentuada, enquanto a demanda residencial de energia elétrica aumentou, assim como a renda. Já no Painel (b), para o setor comercial, é possível observar que a demanda de energia elétrica aumentou, assim como a renda, e que houve uma pequena queda na tarifa de energia elétrica e no preço dos eletrointensivos, sendo neste último mais acentuada nos anos finais. No Painel (c), referente ao setor industrial, nota-se um aumento bastante significativo no PIB quando ocorreram as mudanças na moeda brasileira, fenômeno que também ocorreu no setor comercial. Também é possível observar aumento gradual na demanda industrial de energia elétrica. A variável tarifa da energia elétrica apresentou queda, mas com um leve aumento no final do período, enquanto o preço dos eletrointensivos apresentou queda, sendo 
essa mais acentuada nos anos finais. Já o preço do substituto de energia elétrica cresce até os anos iniciais da década de 80, decresce até 1996 e depois volta a crescer.

Figura 1

Comportamento das séries temporais deflacionadas: (a) demanda residencial; (b) demanda comercial; e (c) demanda industrial de energia elétrica/RS, 1971-2010

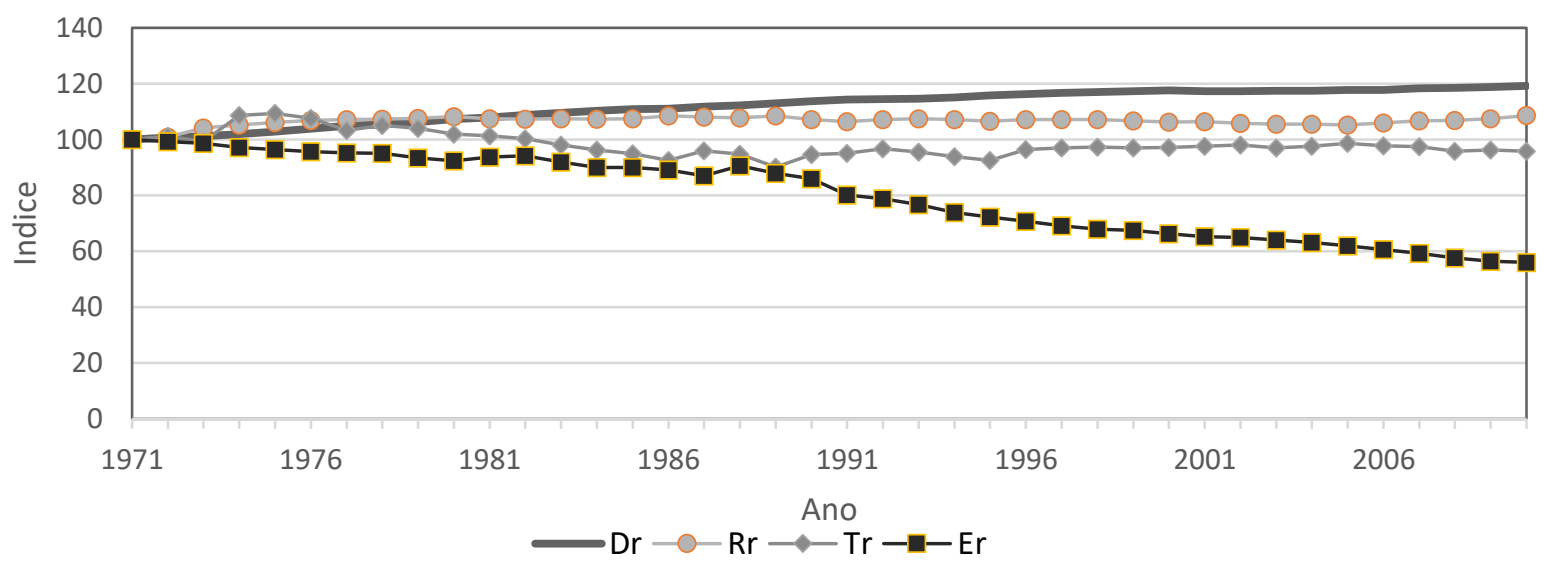

(a)

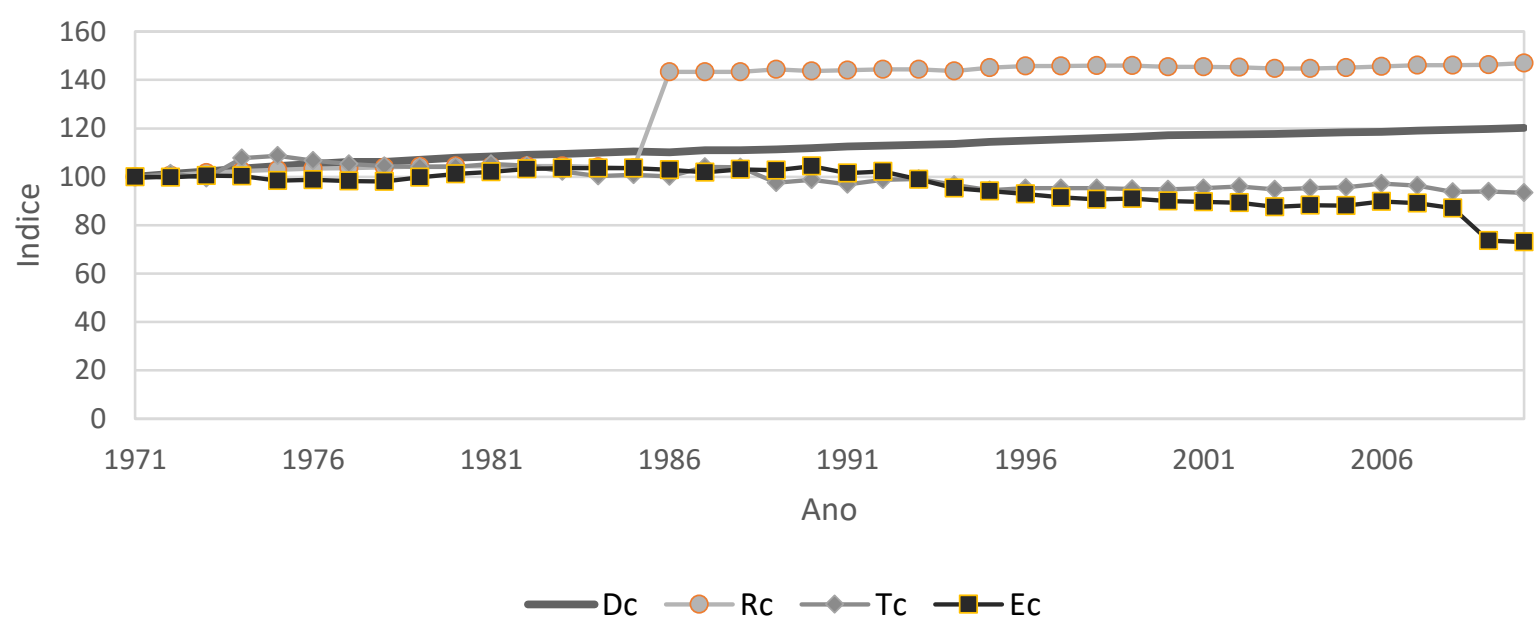

(b) 


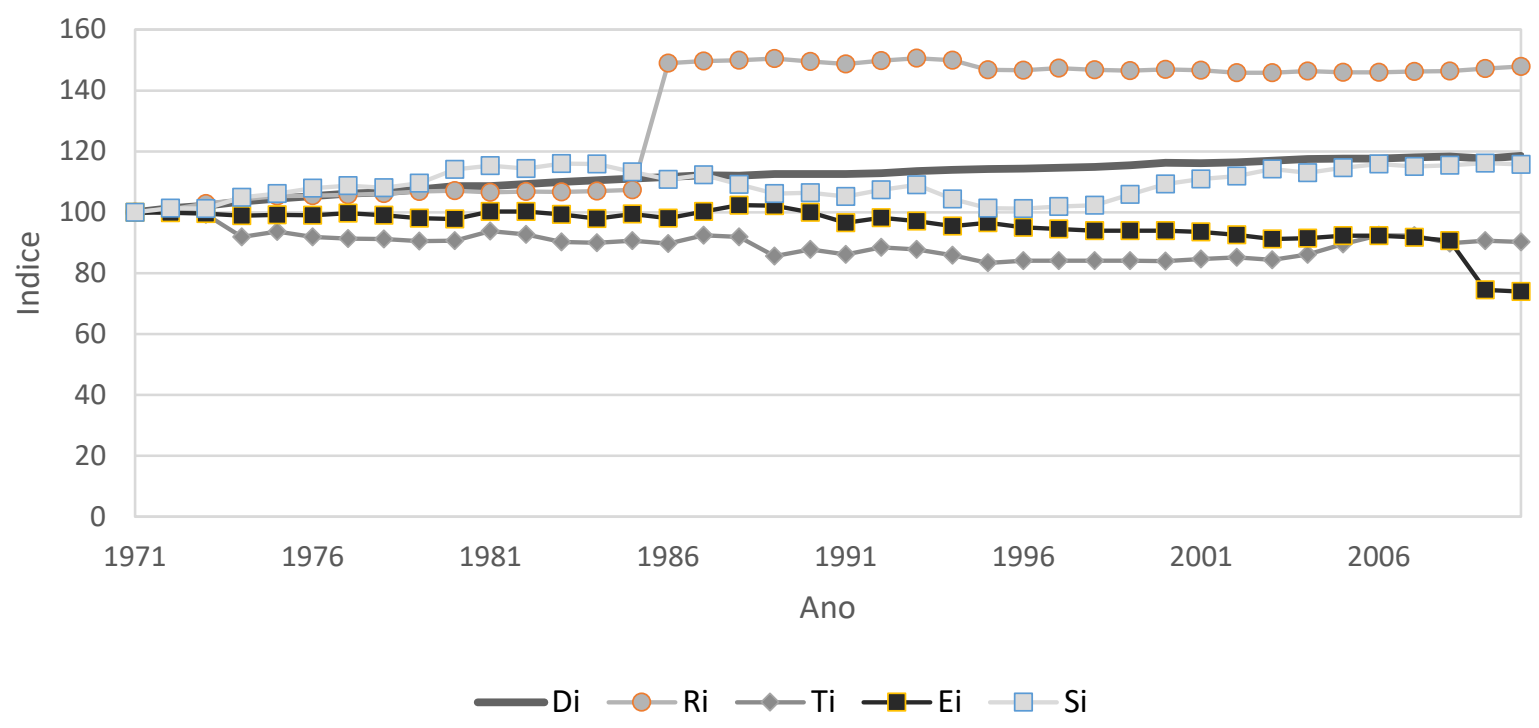

(c)

Fonte: Dados de Eletrobras (2018). Nota: valores relativos ao observado em 1971, considerado igual a 100.

Na sequência, foram realizados os testes ADF e KPSS, sendo avaliada a estacionariedade em cada série, nos três setores estudados, condição necessária para a realização dos testes de cointegração. Constatou-se que todas as variáveis podem ser consideradas estacionárias para a primeira diferença.

O número de defasagens a ser considerado nos modelos estudados, a presença de termos determinísticos (constante e tendência) e de variáveis dummy (Quadro 2) foi determinada pelos critérios de informação de $\mathrm{AIC}, \mathrm{BIC}$ e HQ. Basicamente, o modelo que retorna os menores valores desses critérios é selecionado como o melhor para descrever a série. O acréscimo de variáveis dummy foi testado individualmente e em dupla. Os resultados mais relevantes estão apresentados na Tabela 1, donde se conclui que o modelo mais parcimonioso para o setor residencial não utiliza variáveis dummy, apresentando constante e tendência determinística, além de utilizar apenas uma defasagem. Já para o setor comercial o modelo mais parcimonioso foi o que utilizou duas variáveis dummy (DYc3 e DYc6), com constante e com tendência determinística, além de uma defasagem. No setor industrial, o modelo mais parcimonioso apresenta uma variável dummy (DYi3), constante e tendência determinística, além de utilizar uma defasagem. 


\section{Tabela 1}

Seleção de parâmetros do modelo VAR para a modelagem da demanda residencial, demanda comercial e demanda industrial de energia elétrica/RS, 1971-2010

\begin{tabular}{|c|c|c|c|c|c|c|}
\hline Setor & Variáveis & $\begin{array}{l}\text { Termos da } \\
\text { Equação }\end{array}$ & Defasagens & AIC & $\mathrm{BIC}$ & $\mathrm{HQ}$ \\
\hline \multirow[t]{3}{*}{ Residencial } & $\mathrm{Dr} ; \mathrm{Rr} ; \mathrm{Tr} ; \mathrm{Er}$ & $\begin{array}{l}\text { Constante e } \\
\text { Tendência }\end{array}$ & 1 & $-3,818$ & $-2,773$ & $-3,450$ \\
\hline & $\mathrm{Dr} ; \mathrm{Rr} ; \mathrm{Tr} ; \mathrm{Er} ; \mathrm{DYr} 1$ & $\begin{array}{l}\text { Constante e } \\
\text { Tendência }\end{array}$ & 1 & $-3,193$ & $-1,986$ & $-2,763$ \\
\hline & $\mathrm{Dr} ; \mathrm{Rr} ; \mathrm{Tr} ; \mathrm{Er} ; \mathrm{DYr} 2$ & Constante & 1 & $-3,311$ & $-2,277$ & $-2,944$ \\
\hline \multirow[t]{3}{*}{ Comercial } & $\begin{array}{l}\mathrm{Dc} ; \mathrm{Rc} ; \mathrm{Tc} ; \mathrm{Ec} ; \mathrm{DYc} 3 ; \\
\text { DYc6 }\end{array}$ & $\begin{array}{l}\text { Constante e } \\
\text { Tendência }\end{array}$ & 1 & $-9,775$ & $-8,396$ & $-9,284$ \\
\hline & Dc; Rc; Tc; Ec; DYc3 & $\begin{array}{l}\text { Constante e } \\
\text { Tendência }\end{array}$ & 1 & $-8,770$ & $-7,563$ & $-8,341$ \\
\hline & Dc; Rc; Tc; Ec; DYc6 & Constante & 1 & $-4,826$ & $-3,792$ & $-4,458$ \\
\hline \multirow[t]{3}{*}{ Industrial } & Di; Ri; Ti; Ei; DYi3 & $\begin{array}{l}\text { Constante e } \\
\text { Tendência }\end{array}$ & 1 & $-7,299$ & $-5,920$ & $-6,808$ \\
\hline & Di; Ri; Ti; Ei; DYi6 & $\begin{array}{l}\text { Constante e } \\
\text { Tendência }\end{array}$ & 1 & $-4,763$ & $-3,384$ & $-4,272$ \\
\hline & Di; Ri; Ti; Ei; DYi4 & $\begin{array}{l}\text { Constante e } \\
\text { Tendência }\end{array}$ & 2 & $-4,253$ & $-2,184$ & $-3,517$ \\
\hline
\end{tabular}

Fonte: Elaborada pelos Autores.

Posteriormente, foi aplicado o teste de cointegração de Johansen, sendo executados os testes do traço e do máximo autovalor, de forma a identificar existência de relação de longo prazo entre as variáveis analisadas. Nestes foram consideradas as seguintes situações: sem constante, com constante apenas no vetor de cointegração (restrita), com constante fora do vetor de cointegração (irrestrita), com tendência restrita e com tendência irrestrita. Os resultados obtidos para o teste do traço e o teste do máximo autovalor para o modelo para o setor residencial utilizou constante irrestrita, para o setor comercial o modelo usou tendência irrestrita e para o setor industrial um modelo sem constante. Estes resultados podem ser visualizados na Tabela 2. 
Tabela 2

Testes de Johansen para os modelos de demanda residencial, comercial e industrial de energia elétrica/RS, $1971-2010$

\begin{tabular}{|c|c|c|c|c|c|c|c|}
\hline \multirow{3}{*}{ Teste } & & \multicolumn{3}{|c|}{ Teste traço } & \multicolumn{3}{|c|}{ Teste do máximo autovalor } \\
\hline & & Hipótese & Hipótese & Kalor & Hipótese & Hipótese & Valor \\
\hline & & nula $\left(H_{0}\right)$ & alternativa $\left(H_{1}\right)$ & S & nula $\left(H_{0}\right)$ & alternativa $\left(H_{1}\right)$ & Sotots \\
\hline \multirow{2}{*}{ Residencial } & 10 & $r=0$ & $r>0$ & 0,0022 & $r=0$ & $r=1$ & 0,0082 \\
\hline & 20 & $r \leq 1$ & $r>1$ & 0,0441 & $r=1$ & $r=2$ & 0,2026 \\
\hline \multirow{2}{*}{ Comercial } & 10 & $r=0$ & $r>0$ & 0,0000 & $r=0$ & $r=1$ & 0,0000 \\
\hline & 2o & $r \leq 1$ & $r>1$ & 0,1350 & $r=1$ & $r=2$ & 0,0465 \\
\hline \multirow{2}{*}{ Industrial } & 10 & $r=0$ & $r>0$ & 0,0000 & $r=0$ & $r=1$ & 0,0000 \\
\hline & 20 & $r \leq 1$ & $r>1$ & 0,4714 & $r=1$ & $r=2$ & 0,3094 \\
\hline
\end{tabular}

Fonte: Elaborada pelos Autores.

Nos três setores consumidores, o primeiro teste traço encontrou evidências de que existe pelo menos um vetor de cointegração entre as variáveis, enquanto o segundo indicou a existência de no máximo um vetor de cointegração entre as variáveis. Já para o teste do máximo autovalor, o primeiro rejeita a inexistência de vetor de cointegração, enquanto o segundo não rejeita a existência de um vetor de cointegração. Deste modo, as séries são cointegráveis, sugerindo a existência de relação de longo prazo entre elas.

$$
\begin{aligned}
& D_{r}=0,17141 R_{r}-4,1806 T_{r}-2,9280 E_{r} \\
& D_{c}=0,88702 R_{c}-0,10373 T_{c}+0,1803 E_{c} \\
& D_{i}=1,1420 R_{i}-0,70779 T_{i}-1,4288 E_{i}
\end{aligned}
$$

A análise de resíduos dos modelos mostrou que eles podem ser considerados independentes (Ljung-Box residencial: valor $p=0,2856$; Ljung-Box comercial: valor $p=0$, 9360; Ljung-Box industrial: valor $p=0,6674$ ), normalmente distribuídos (Jarque-Bera residencial: valor $p=0,7674$; Jarque Bera comercial: valor $p=0$, 4062; Jarque Bera industrial: valor $p=0,2483$ ) e homocedásticos (ARCH residencial: valor $p=0,0524$; $A R C H$ comercial: valor $p=0,1207$; $A R C H$ industrial: valor $p=0,2067$ ).

Por meio das Equações 5, 6 e 7, é possível verificar que as varáveis renda e tarifa se comportam como o esperado pela teoria econômica e trabalhos como Schmidt e Lima (2004), Mattos (2004), Viana (2010) e Silveira (2017), em todas as equações. Sendo assim, a variável renda tem uma relação direta 
com a demanda e a tarifa uma relação inversa. O mesmo ocorre com as variáveis preço dos eletrodomésticos e preço das máquinas e equipamentos, que possuem uma relação inversa à demanda. No entanto, para o setor comercial, a variável preço dos eletrointensivos apresentou relação direta com a demanda comercial de energia elétrica, diferentemente do esperado.

Na sequência, foi realizada a correção dos modelos anteriores por meio do modelo vetorial de correção de erros (VEC).

$$
\begin{aligned}
& \Delta D_{r t}=0,4025-0,0081\left(u_{t-1}\right) \\
& \Delta D_{c t}=0,1072 D Y_{c 3}+0,0215 D Y_{c 6}-0,0169\left(u_{t-1}\right) \\
& \Delta D_{i t}=0,0063 S_{i}+0,0553 D Y_{c 3}-0,0016\left(u_{t-1}\right)
\end{aligned}
$$

Os modelos apresentados nas Equações 8, 9 e 10 exibiram respectivamente um coeficiente de determinação $R^{2}$ de 0,5407, 0,4897 e 0,7115 e $R^{2}$-ajustado de 0,5283, 0,4297 e 0,6955 indicando que alterações nas variáveis consideradas explicam 54,07\%, 48,97\% e 71,15\% das variações da demanda residencial, comercial e industrial de energia elétrica, respectivamente. O coeficiente do termo erro das equações acima indica para o setor residencial, comercial e industrial que respectivamente 0,81\%, $1,69 \%$ e $0,16 \%$ da diferença entre a relações de longo e curto prazo é corrigida a cada ano.

A análise dos resíduos dos modelos vetorial de correção de erros mostraram que eles continuam sendo considerados independentes(Ljung-Box residencial: valor $p=0,6807$, Ljung-Box comercial: valor $p=0,9150$ e Ljung-Box industrial: valor $p=0,9029$ ), normalmente distribuídos (Jarque Bera residencial: valor $p=0,6187$, Jarque Bera comercial: valor $p=0,2839$ e Jarque Bera industrial: valor $p=0,4172$ ) e homocedásticos (ARCH residencial: valor $p=0,1321, A R C H$ comercial: valor $p=0,1270$ e ARCH industrial: valor $p=0,2302$ ).

Na validação dos modelos para a previsão das demandas residencial, comercial e industrial de energia elétrica, os indicadores utilizados foram o erro absoluto médio (MAE), raiz do erro quadrático médio (RMSE) e erro absoluto percentual médio (MAPE), além da estatística $U$ de Theil. As medidas encontradas estão apresentadas na Tabela 3, sendo identificado o modelo VAR sem correção como aquele de melhor desempenho para o setor residencial e o modelo VAR-VEC para ambos os setores comercial e industrial. 


\section{Tabela 3}

Indicadores da precisão da previsão dos modelos VAR e VAR_VEC para a demanda residencial, demanda comercial e demanda industrial de energia elétrica/RS, 2011-17

\begin{tabular}{clcccc}
\hline \multirow{2}{*}{ Setor } & \multicolumn{1}{c}{ Modelo } & RMSE & MAE & MAPE (\%) & U de Theil \\
\multirow{2}{*}{ Residencial } & VAR & $570.437,92$ & $481.960,55$ & 5,9077 & 0,0722 \\
& VAR-VEC & $871.697,50$ & $774.033,87$ & 9,5082 & 0,1103 \\
\hline \multirow{2}{*}{ Comercial } & VAR & $524.245,26$ & $382.753,40$ & 7,5725 & 0,1037 \\
& VAR-VEC & $422.328,15$ & $365.169,84$ & 7,1106 & 0,0836 \\
\hline \multirow{2}{*}{ Industrial } & VAR & $3.862 .966,13$ & $3.197 .038,98$ & 32,215 & 0,2321 \\
& VAR-VEC & $2.283 .083,86$ & $1.851 .625,98$ & 18,702 & 0,4635
\end{tabular}

Fonte: Elaborada pelos Autores.

\subsection{Modelo ARIMA}

A análise dos gráficos em linha das demandas residencial, comercial e industrial de energia elétrica sugeriu, em todas as séries, a aplicação da transformação logarítmica com o intuito de reduzir a variabilidade. A análise da estacionariedade do logaritmo destes dados em nível (FAC, FACP, ADF e KPSS) indicou a necessidade de utilização da primeira diferença para todos os modelos. As Figuras 2, 3 e 4 mostram os gráficos em linhas e da FAC e FACP da primeira diferença do logaritmo dos dados para as demandas residencial, comercial e industrial respectivamente, que podem ser considerados estacionários de acordo com os testes ADF (valor $p<0,01$ ) e KPSS (valor $p>0,10$ ). Os gráficos da FAC e da FACP para as demandas residencial e comercial, apresentam uma queda lenta com muitas correlações significativas e uma queda abrupta na primeira defasagem, sugerindo um modelo ARIMA $(1,1,0)$. Pela subjetividade da interpretação gráfica, admitiram-se as variações de \pm 1 na ordem do componente autorregressivo e de +1 na ordem do componente de médias móveis. Os modelos considerados como candidatos para os setores residencial e comercial foram: ARIMA $(0,1,0)$, ARIMA $(1,1,0)$, ARIMA $(2,1,0)$, ARIMA $(0,1,1)$, ARIMA $(1,1,1)$, ARIMA $(2,1,1)$. Para a demanda industrial, os gráficos da FAC e da FACP, sugerem o modelo ARIMA $(0,1,0)$. Para aumentar as possibilidades na análise, foi admitido variações de até +2 na ordem dos componentes auto regressivos e de médias móveis e os seguintes modelos candidatos foram elencados: ARIMA $(0,1,0)$, ARIMA $(0,1,1), \operatorname{ARIMA}(0,1,2), A R I M A$ $(1,1,0), \operatorname{ARIMA}(1,1,1), \operatorname{ARIMA}(1,1,2)$, ARIMA $(2,1,0), \operatorname{ARIMA}(2,1,1)$ e ARIMA $(2,1,2)$. 
Figura 2

(a) Primeira diferença do logaritmo da demanda residencial, (b) correlogramas da FAC e

FACP da demanda residencial

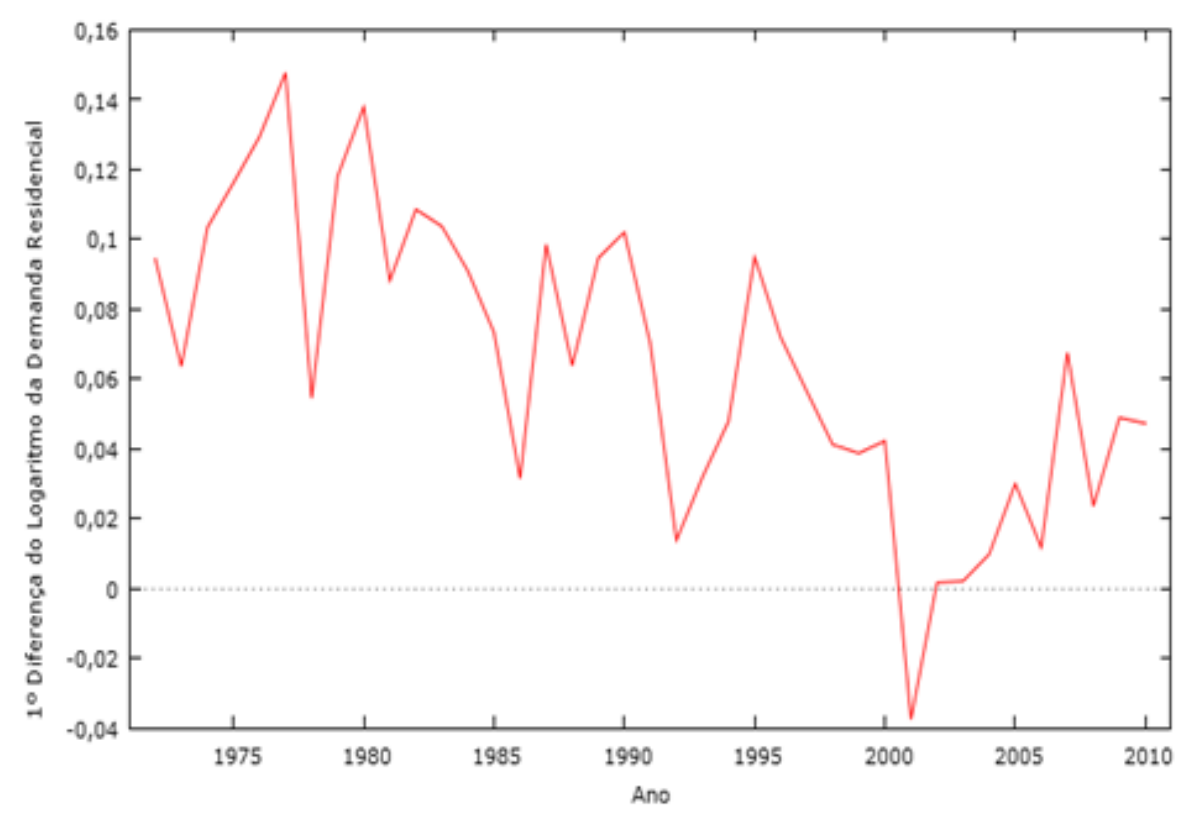

(a)
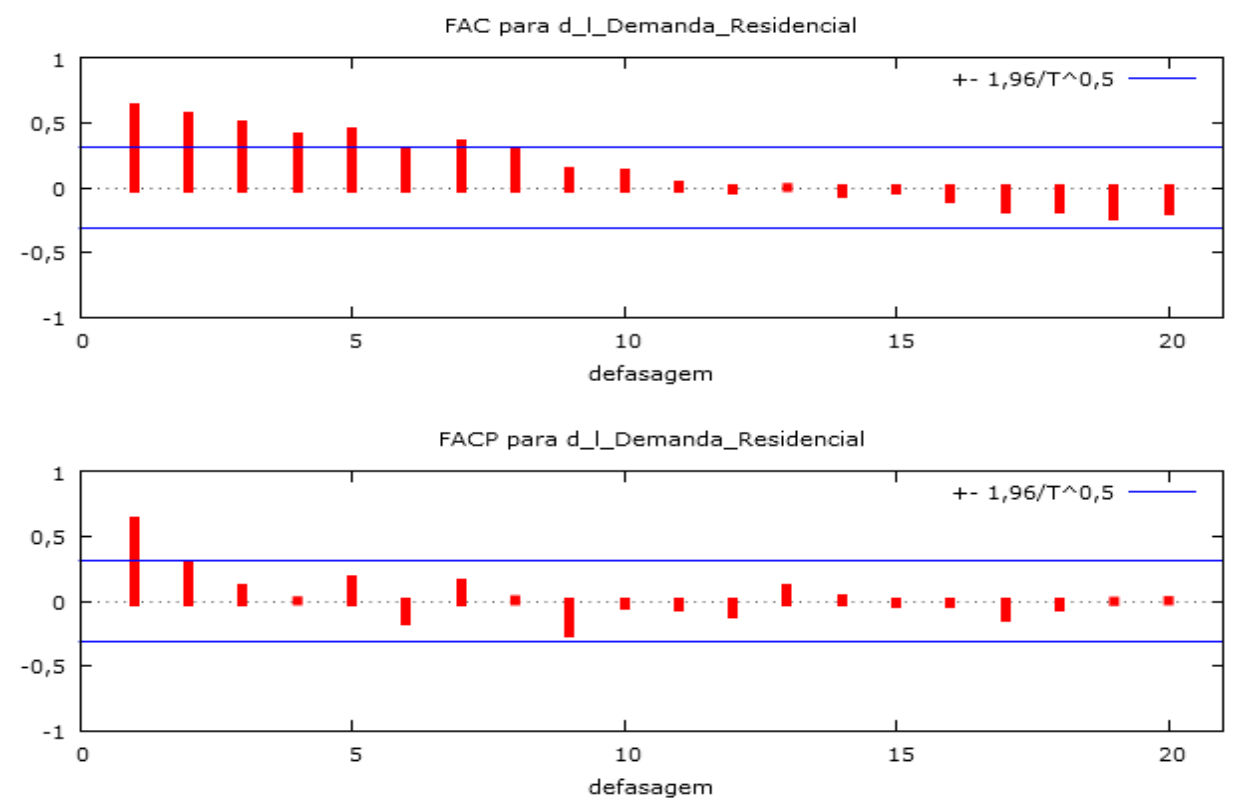

(b)

Fonte: Elaborada pelos Autores. 
Figura 3

(a) Primeira diferença do logaritmo da demanda comercial, (b) correlogramas da FACe

FACP da demanda comercial

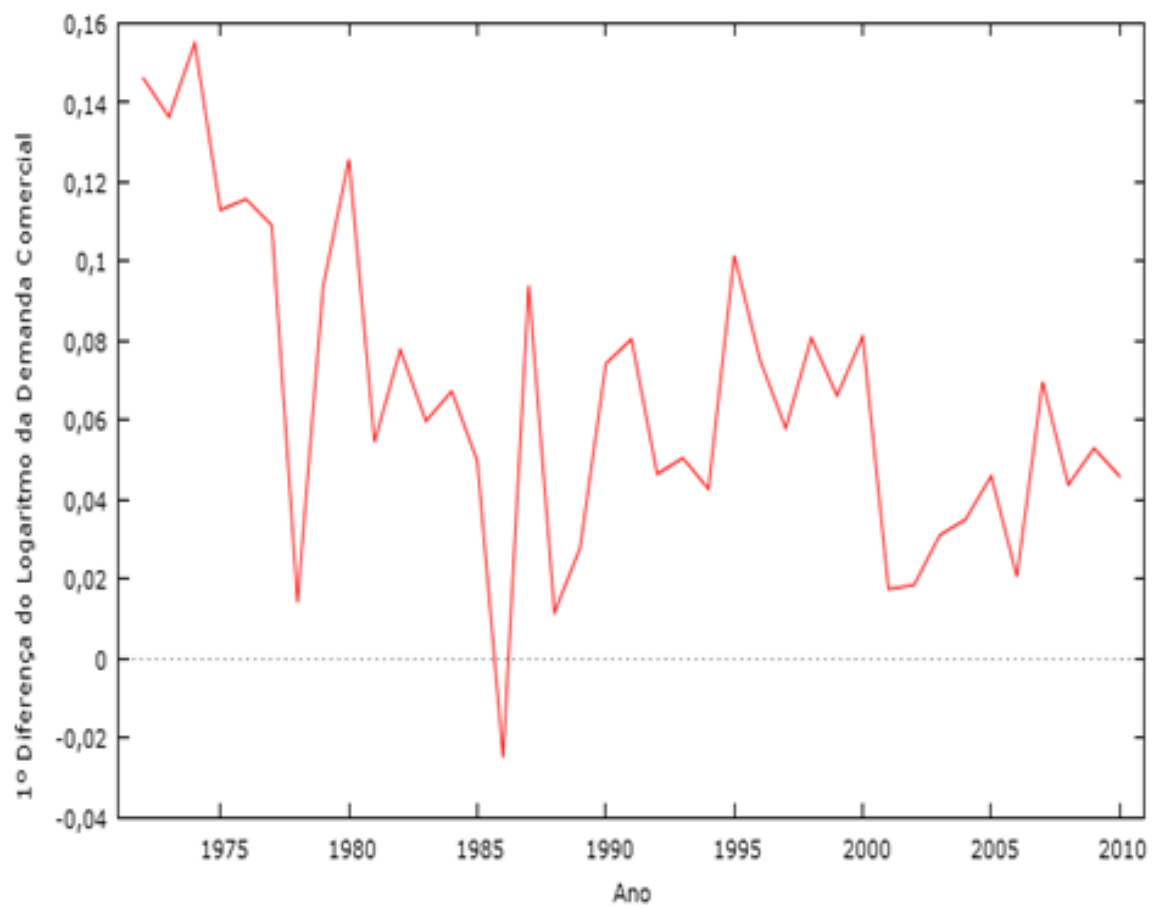

(a)

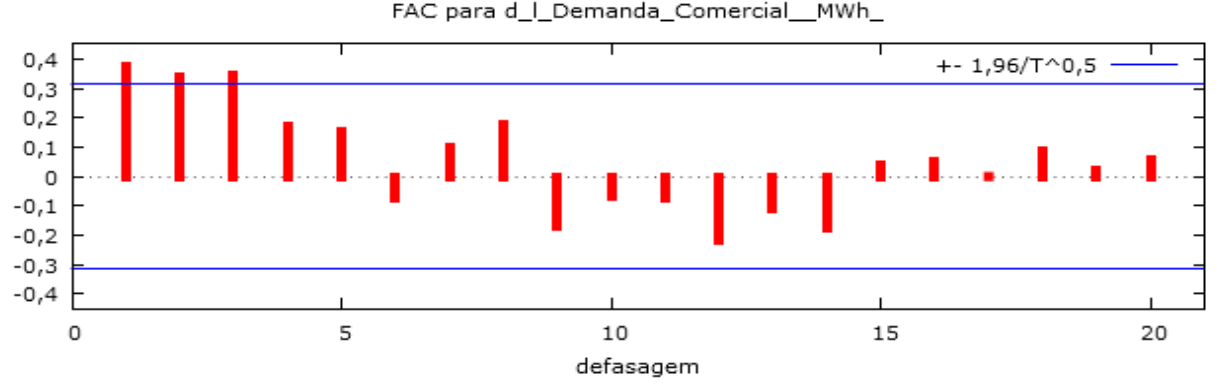

FACP para d_I_Demanda_Comercial__MWh_

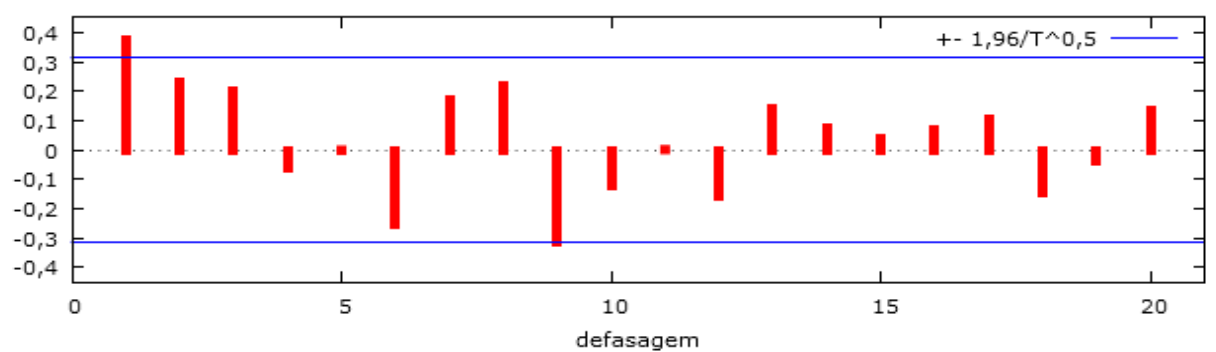

(b)

Fonte: Elaborada pelos Autores. 
Figura 4

(a) Primeira diferença do logaritmo da demanda industrial, e (b) correlogramas da

\section{FAC e FACP da demanda industrial}

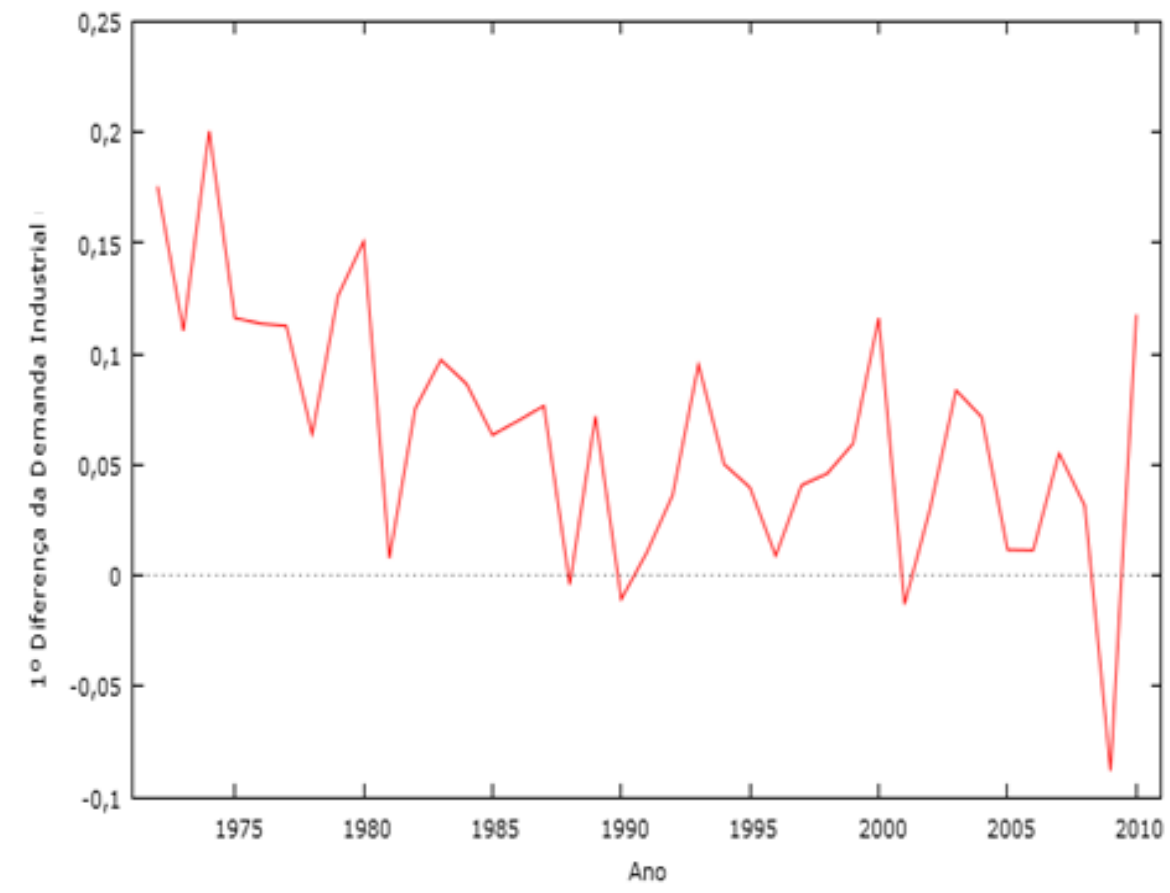

(a)
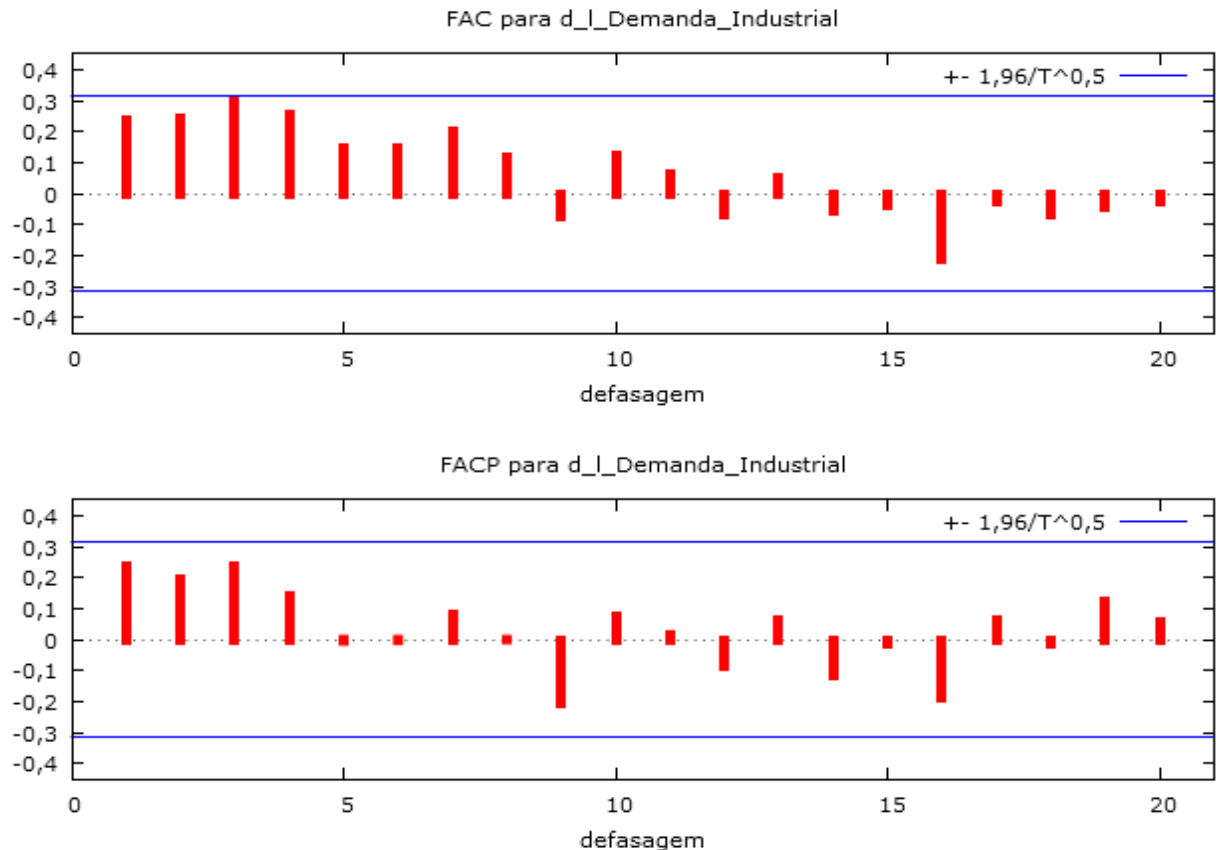

(b)

Fonte: Elaborada pelos Autores. 
Na seleção do modelo pelos critérios de informação, foi considerado que o modelo mais parcimonioso para o setor residencial foi o ARIMA $(1,1,1)$ sem constante, seguido pelos modelos ARIMA $(1,1,1)$ com constante, ARIMA $(2,1,0)$ com constante e ARIMA $(1,1,0)$ sem constante. Para o setor comercial, o modelo mais parcimonioso foi o modelo ARIMA $(1,1,1)$ sem constante, seguido pelos modelos ARIMA $(1,1,1)$ com constante, ARIMA $(1,1,0)$ com constante e ARIMA $(2,1,0)$ sem constante. Já para o setor industrial, o modelo mais parcimonioso foi o ARIMA $(1,1,2)$ sem constante, seguido pelos modelos ARIMA $(1,1,1)$ sem constante e com constante, e do modelo ARIMA $(2,1,0)$ sem constante. Estes resultados podem ser visualizados na Tabela 4. Nesta análise, foram desconsiderados os modelos com coeficientes estatisticamente não significativos (valor $p>0,05$ ).

\section{Tabela 4}

Resultados dos critérios de informação nos modelos candidatos ARIMA $(p, 1, q)$ para a demanda residencial, demanda comercial e demanda industrial de energia elétrica/RS, 1971-2010

\begin{tabular}{|c|c|c|c|c|c|}
\hline \multirow{2}{*}{ Setor } & \multirow{2}{*}{ Modelo } & \multicolumn{4}{|c|}{ Critério de informação } \\
\hline & & Constante & Schwarz & Akaike & Hannan-Quinn \\
\hline \multirow{4}{*}{ Residencial } & $(1,1,1)$ & Sem & $-145,838$ & $-150,829$ & $-149,038$ \\
\hline & $(1,1,1)$ & Com & $-145,227$ & $-151,882$ & $-149,494$ \\
\hline & $(2,1,0)$ & Com & $-144,298$ & $-150,953$ & $-148,565$ \\
\hline & $(1,1,0)$ & Com & $-144,824$ & $-149,814$ & $-148,024$ \\
\hline \multirow{4}{*}{ Comercial } & $(1,1,1)$ & Sem & $-139,562$ & $-144,552$ & $-142,762$ \\
\hline & $(1,1,1)$ & Com & $-138,381$ & $-145,035$ & $-142,648$ \\
\hline & $(1,1,0)$ & Com & $-137,124$ & $-142,115$ & $-140,324$ \\
\hline & $(2,1,0)$ & Sem & $-135,181$ & $-140,171$ & $-138,381$ \\
\hline \multirow{4}{*}{ Industrial } & $(1,1,2)$ & Sem & $-109,786$ & $-116,440$ & $-114,053$ \\
\hline & $(1,1,1)$ & Sem & $-110,104$ & $-115,095$ & $-113,304$ \\
\hline & $(1,1,1)$ & Com & $-108,419$ & $-115,073$ & $-112,686$ \\
\hline & $(2,1,0)$ & Sem & $-102,179$ & $-107,170$ & $-105,379$ \\
\hline
\end{tabular}

Fonte: Elaborada pelos Autores.

Os resultados dos testes Ljung-Box, Jarque-Bera e ARCH, mostram que todos os modelos selecionados cumprem todas as suposições do modelo teórico, a um nível de significância de 0,05.

Na validação dos modelos candidatos, foram encontradas as medidas apresentadas na Tabela 5 , sendo identificado o modelo ARIMA $(2,1,0)$ sem constante como o de melhor desempenho para prever a demanda residencial de energia elétrica/RS, embora o modelo ARIMA $(1,1,1)$ sem constante tenha obtidos indicadores bastante próximos. Já para prever a demanda comercial de energia elétrica/RS, foi identificado o modelo ARIMA $(2,1,0)$ sem constante. Enquanto que o modelo de melhor 
desempenho para prever a demanda industrial de energia elétrica/RS foi o ARIMA $(1,1,2)$ sem constante.

\section{Tabela 5}

Capacidade preditiva dos modelos candidatos $\operatorname{ARIMA}(p, 1, q)$ para a demanda residencial, demanda comercial e demanda industrial de energia elétrica/RS, 2011-17

\begin{tabular}{ccccccc}
\multirow{2}{*}{ Setor } & ARIMA & Constante & RMSE & MAE & MAPE $(\%)$ & U de Theil \\
& $(p, 1, q)$ & & & & \\
\multirow{2}{*}{ Residencial } & $(2,1,0)$ & Sem & $312.044,91$ & $247.507,06$ & 3,018 & 0,002 \\
& $(1,1,1)$ & Sem & $326.523,31$ & $251.559,24$ & 3,058 & 0,002 \\
& $(1,1,1)$ & Com & $719.274,30$ & $527.826,08$ & 6,464 & 0,091 \\
& $(1,1,0)$ & Com & $1.044 .827,86$ & $757.466,29$ & 9,319 & 0,132 \\
\hline \multirow{3}{*}{ Comercial } & $(2,1,0)$ & Sem & $362.076,52$ & $333.726,29$ & 6,590 & 0,005 \\
& $(1,1,1)$ & Sem & $405.825,70$ & $353.297,29$ & 7,001 & 0,080 \\
& $(1,1,1)$ & Com & $595.718,89$ & $463.315,83$ & 9,197 & 0,118 \\
& $(1,1,0)$ & Com & $745.851,71$ & $525.037,73$ & 10,433 & 0,148 \\
& $(1,1,2)$ & Sem & $867.030,11$ & $667.835,69$ & 6,763 & 0,085 \\
& $(2,1,0)$ & Sem & $1.444 .601,55$ & $1.188 .074,21$ & 12,002 & 0,141 \\
& $(1,1,1)$ & Sem & $1.829 .996,53$ & $1.478 .264,39$ & 14,944 & 0,179 \\
& $(1,1,1)$ & Com & $2.533 .940,38$ & $2.047 .106,44$ & 20,678 & 0,249
\end{tabular}

Fonte: Elaborada pelos Autores.

\subsection{Avaliação da capacidade preditiva}

Nesta seção, avaliaram-se as capacidades preditivas dos modelos selecionados pelos métodos VAR, VAR-VEC e ARIMA para diferentes horizontes de previsão, para as demandas residencial, comercial e industrial.

No setor residencial, é possível constatar, por meio da Tabela 6, que o modelo VAR obteve o melhor resultado para previsão um passo à frente (MAPE $=0,40 \%)$, mas foi suplantado pelo modelo ARIMA $(2,1,0)$ nos demais horizontes de previsão, sendo este modelo identificado como aquele com melhor capacidade preditiva para até sete passos à frente, com um MAPE máximo de 3,02\%. Este modelo foi o único que conseguiu satisfazer as condições impostas em contratos de fornecimento de energia elétrica para quase todo o horizonte de previsão considerado neste estudo, pois de acordo com Cabral et al. (2017), as empresas fornecedoras de energia elétrica devem fazer previsões tolerando um erro máximo de 3\% para estarem isentas de multas contratuais. 


\section{Tabela 6}

Capacidade preditiva dos modelos VAR, VAR-VEC e ARIMA $(2,1,0)$ para a demanda residencial de energia elétrica/RS, 2011-17

\begin{tabular}{|c|c|c|c|c|}
\hline Modelo & Horizonte de previsão & MAE & RMSE & MAPE (\%) \\
\hline \multirow{7}{*}{$V A R$} & 2011 & 28444,0 & 28444,0 & 0,40 \\
\hline & 2011-12 & 134063,0 & 170670,0 & 1,84 \\
\hline & 2011-13 & 252842,7 & 315568,6 & 3,33 \\
\hline & 2011-14 & 464474,8 & 613874,4 & 5,73 \\
\hline & 2011-15 & 468713,6 & 590464,5 & 5,79 \\
\hline & 2011-16 & 483842,2 & 585415,1 & 5,95 \\
\hline & 2011-17 & 481960,9 & 570438,2 & 5,91 \\
\hline \multirow{7}{*}{$V A R-V E C$} & 2011 & 91611,0 & 91611,0 & 1,32 \\
\hline & 2011-12 & 231220,5 & 270099,5 & 3,18 \\
\hline & 2011-13 & 385549,7 & 457468,5 & 5,11 \\
\hline & 2011-14 & 634359,3 & 795991,2 & 7,88 \\
\hline & 2011-15 & 677489,4 & 807084,2 & 8,42 \\
\hline & 2011-16 & 733319,5 & 844789,9 & 9,05 \\
\hline & 2011-17 & 774033,9 & 871697,5 & 9,51 \\
\hline \multirow{7}{*}{$\operatorname{ARIMA}(2,1,0)$} & 2011 & 104234,1 & 104234,1 & 1,50 \\
\hline & 2011-12 & 66561,6 & 76483,1 & 0,95 \\
\hline & 2011-13 & 72457,5 & 79156,6 & 0,99 \\
\hline & 2011-14 & 192830,1 & 285331,1 & 2,37 \\
\hline & 2011-15 & 194231,8 & 270403,7 & 2,39 \\
\hline & 2011-16 & 206249,3 & 269730,2 & 2,53 \\
\hline & 2011-17 & 247507,1 & 312044,9 & 3,02 \\
\hline
\end{tabular}

Fonte: Elaborada pelos Autores.

Para a demanda comercial, Tabela 7, é possível constatar que o modelo VAR obteve os melhores resultados para previsões até seis passos à frente, mas foi suplantado pelo modelo ARIMA $(2,1,0)$ para previsão sete passos à frente, sendo este modelo identificado como aquele com melhor capacidade preditiva para sete unidades de tempo a frente, com um MAPE de 6,59\%. Este modelo, entretanto, não conseguiu satisfazer as condições impostas em contratos de fornecimento de energia elétrica para nenhum dos horizontes de previsão considerados neste estudo. Já o modelo VAR conseguiu satisfazer esta condição para previsões a até quatro passos à frente. 


\section{Tabela 7}

Capacidade preditiva dos modelos VAR, VAR_VEC e ARIMA $(2,1,0)$ para a demanda comercial de energia elétrica/RS, 2011-17

\begin{tabular}{|c|c|c|c|c|}
\hline Modelo & Horizonte de previsão & MAE & RMSE & MAPE (\%) \\
\hline \multirow{7}{*}{ VAR } & 2011 & 84290,0 & 84290,0 & 1,83 \\
\hline & 2011-12 & 150070,0 & 163853,6 & 3,09 \\
\hline & $2011-13$ & 112940,3 & 135637,1 & 2,31 \\
\hline & 2011-14 & 134829,0 & 154426,7 & 2,65 \\
\hline & 2011-15 & 166018,8 & 189706,3 & 3,23 \\
\hline & 2011-16 & 270666,0 & 367473,9 & 5,34 \\
\hline & $2011-17$ & 382753,3 & 524245,4 & 7,57 \\
\hline \multirow{7}{*}{$V A R-V E C$} & 2011 & 200897,0 & 200897,0 & 4,36 \\
\hline & 2011-12 & 332115,0 & 357097,4 & 6,84 \\
\hline & 2011-13 & 365462,3 & 383751,9 & 7,42 \\
\hline & 2011-14 & 463358,8 & 503715,8 & 9,03 \\
\hline & 2011-15 & 460205,8 & 493002,8 & 8,94 \\
\hline & 2011-16 & 407920,3 & 454004,2 & 7,94 \\
\hline & 2011-17 & 365169,7 & 422328,1 & 7,11 \\
\hline \multirow{7}{*}{$\operatorname{ARIMA}(2,1,0)$} & 2011 & 228236,6 & 228236,6 & 4,96 \\
\hline & 2011-12 & 317111,7 & 329330,6 & 6,56 \\
\hline & 2011-13 & 304665,4 & 313682,5 & 6,22 \\
\hline & 2011-14 & 354318,4 & 370296,5 & 6,97 \\
\hline & 2011-15 & 300194,8 & 333311,8 & 5,90 \\
\hline & 2011-16 & 306058,0 & 333656,4 & 6,03 \\
\hline & 2011-17 & 333726,3 & 362076,5 & 6,59 \\
\hline
\end{tabular}

Fonte: Elaborada pelos Autores.

Na sequência, para a demanda industrial, a Tabela 8 mostra que o modelo VAR-VEC obteve o melhor resultado para previsão um passo à frente (MAPE $=0,19 \%$ ), mas foi suplantado pelo modelo ARIMA $(1,1,2)$ para os demais horizontes de previsão. Este modelo, entretanto, conseguiu satisfazer as condições impostas em contratos de fornecimento de energia elétrica apenas até quatro passos à frente. 
Tabela 8

Capacidade preditiva dos modelos VAR, VAR_VEC e ARIMA $(1,1,2)$ para a demanda industrial de energia elétrica/RS, 2011-17

\begin{tabular}{|c|c|c|c|c|}
\hline Modelo & Horizonte de previsão & MAE & RMSE & MAPE (\%) \\
\hline \multirow{7}{*}{$V A R$} & 2011 & 224212,0 & 224212,0 & 2,11 \\
\hline & 2011-12 & 738631,5 & 900113,3 & 7,17 \\
\hline & 2011-13 & 1047984 & 1210821 & 9,99 \\
\hline & 2011-14 & 1483245 & 1744774 & 14,15 \\
\hline & 2011-15 & 2053356 & 2488322 & 20,09 \\
\hline & 2011-16 & 2666137 & 3260678 & 26,80 \\
\hline & 2011-17 & 3194568 & 3860188 & 32,19 \\
\hline \multirow{7}{*}{ VAR-VEC } & 2011 & 20267,0 & 20267,0 & 0,19 \\
\hline & 2011-12 & 396434,5 & 546500,0 & 3,86 \\
\hline & 2011-13 & 539302,7 & 652690,3 & 5,15 \\
\hline & 2011-14 & 781522,3 & 942420,3 & 7,47 \\
\hline & 2011-15 & 1146691 & 1438817 & 11,25 \\
\hline & 2011-16 & 1543539 & 1949198 & 15,57 \\
\hline & 2011-17 & 1851626 & 2283084 & 18,70 \\
\hline \multirow{7}{*}{$\operatorname{ARIMA}(1,1,2)$} & 2011 & 527570,2 & 527570,2 & 4,98 \\
\hline & 2011-12 & 265409,1 & 373055,5 & 2,50 \\
\hline & 2011-13 & 253096,0 & 331933,4 & 2,38 \\
\hline & 2011-14 & 227038,0 & 296942,7 & 2,14 \\
\hline & 2011-15 & 366703,4 & 491731,8 & 3,59 \\
\hline & 2011-16 & 557548,9 & 763158,8 & 5,64 \\
\hline & 2011-17 & 667835,7 & 867030,1 & 6,76 \\
\hline
\end{tabular}

Fonte: Elaborada pelos Autores.

Complementando a análise, a Tabela 9 (demanda residencial), Tabela 10 (demanda comercial) e Tabela 11 (demanda industrial) apresentam os valores observados, estimações por ponto e por intervalo com confiança de 0,95 para o modelo com melhor capacidade preditiva, evidenciando que todos contêm os valores observados (reais), embora aqueles construídos a partir do modelo ARIMA apresentem maior amplitude.

\section{Tabela 9}

Modelo ARIMA $(2,1,0)$ sem constante: intervalos de confiança para a previsão da demanda residencial de energia elétrica/RS, 2011-17

\begin{tabular}{cccc}
\hline Ano & Valor observado & Previsão & \multicolumn{1}{|c}{ IC $-95 \%$} \\
2011 & $6.956 .044,0$ & $7.060 .278,1$ & $6.621 .523,3 ; 7.528 .105,7$ \\
2012 & $7.336 .379,0$ & $7.365 .268,0$ & $6.542 .860,4 ; 8.291 .040,2$ \\
2013 & $7.750 .414,0$ & $7.666 .164,6$ & $6.366 .522,8 ; 9.231 .102,1$ \\
2014 & $8.516 .881,0$ & $7.962 .933,3$ & $6.145 .950,7 ; 10.317 .086,9$ \\
2015 & $8.054 .982,7$ & $8.254 .821,4$ & $5.886 .081,2 ; 11.576 .815,6$ \\
2016 & $8.275 .110,0$ & $8.541 .447,1$ & $5.602 .446,9 ; 13.022 .237,1$ \\
2017 & $8.327 .298,0$ & $8.822 .351,5$ & $5.303 .571,4 ; 14.675 .764,2$
\end{tabular}

Fonte: Elaborada pelos Autores. 


\section{Tabela 10}

Modelo ARIMA $(2,1,0)$ sem constante: intervalos de confiança para a previsão da demanda comercial de energia elétrica/RS, 2011-17

\begin{tabular}{cccc} 
Ano & Valor observado & Previsão & IC $-95 \%$ \\
2011 & $4.603 .000,0$ & $4.374 .763,4$ & $4.075 .320,7 ; 4.696 .208,3$ \\
2012 & $4.972 .000,0$ & $4.566 .013,2$ & $4.024 .913,6 ; 5.179 .857,0$ \\
2013 & $5.040 .224,0$ & $4.760 .451,1$ & $3.903 .011,5 ; 5.806 .253,0$ \\
2014 & $5.456 .817,0$ & $4.953 .539,8$ & $3.757 .367,0 ; 6.530 .525,7$ \\
2015 & $5.230 .826,0$ & $5.147 .125,7$ & $3.584 .326,0 ; 7.391 .320,8$ \\
2016 & $5.004 .438,0$ & $5.339 .812,0$ & $3.397 .039,9 ; 8.393 .658,2$ \\
2017 & $5.032 .131,0$ & $5.531 .867,4$ & $3.199 .586,1 ; 9.564 .223,8$ \\
\hline
\end{tabular}

Fonte: Elaborada pelos Autores.

\section{Tabela 11}

Modelo ARIMA $(1,1,2)$ sem constante: intervalos de confiança para a previsão da demanda industrial de energia elétrica/RS, 2011-17

\begin{tabular}{cccc} 
Ano & Valor observado & Previsão & IC $-95 \%$ \\
2011 & $10.595 .000,0$ & $10.067 .429,8$ & $9.175 .862,9 ; 11.045 .614,2$ \\
2012 & $10.248 .000,0$ & $10.251 .247,9$ & $9.058 .490,0 ; 11.601 .059,6$ \\
2013 & $10.665 .149,0$ & $10.436 .679,1$ & $8.857 .313,0 ; 12.297 .665,4$ \\
2014 & $10.474 .837,0$ & $10.623 .700,9$ & $8.598 .196,2 ; 13.126 .360,3$ \\
2015 & $9.886 .935,4$ & $10.812 .300,7$ & $8.298 .522,1 ; 14.087 .564,3$ \\
2016 & $9.490 .690,0$ & $11.002 .466,1$ & $7.970 .246,7 ; 15.188 .270,3$ \\
2017 & $9.864 .617,2$ & $11.194 .173,7$ & $7.622 .248,8 ; 16.439 .951,5$ \\
\hline
\end{tabular}

Fonte: Elaborada pelos Autores.

\section{Considerações finais}

Este estudo buscou modelar a demanda de energia elétrica no estado do Rio Grande do Sul, considerando informações das últimas três décadas, para os três principais setores consumidores: residencial, comercial e industrial, avaliando o possível ganho na precisão das estimativas obtidas pelos modelos VAR, VAR-VEC e ARIMA. Os modelos multivariados VAR e VAR-VEC utilizaram, além dos dados da demanda de energia elétrica, dados da tarifa de energia elétrica, PIB, preços de eletrodomésticos e preço de materiais e equipamentos elétricos, enquanto que o modelo ARIMA utilizou apenas os dados da demanda de energia elétrica.

Ao contrário do estudo desenvolvido por Castro et al. (2016) e diferentemente do esperado, nos três setores consumidores, os melhores modelos foram os obtidos por meio da metodologia de Box-Jenkins para uma previsão sete passos à frente. Os modelos ARIMA, além de apresentarem 
menores valores para as medidas que quantificam os erros de previsão, forneceram intervalos de confiança que contemplam todos os valores observados, embora estes tenham apresentado maior amplitude do que aqueles obtidos pela outra metodologia. Uma outra vantagem deste modelo é que ele precisa de menos informações para ser gerado, pois é univariado, sendo mais facilmente implementado, além de conter uma quantidade menor de possíveis erros de mensuração.

O comportamento da demanda residencial de energia elétrica parece ter sido similar ao comportamento da demanda comercial de energia elétrica, pois os modelos identificados apresentaram a mesma estrutura. No ajuste, o melhor modelo foi ARIMA $(1,1,1)$ sem constante, enquanto para a previsão, o modelo ARIMA $(2,1,0)$ também sem constante. Já para a demanda industrial de energia elétrica, o melhor modelo no ajuste coincidiu com o melhor modelo para a previsão: ARIMA $(1,1,2)$ sem constante. Esses achados vão ao encontro dos resultados obtidos por Amaro et al. (2017), que utilizaram a metodologia de Box-Jenkins para modelar a demanda brasileira de energia elétrica, encontrando modelo de estrutura similar com MAPE $=1,65 \%$ para previsão 50 passos à frente.

É possível que esses resultados tenham sido influenciados por uma aparente mudança no padrão de comportamento das séries na segunda metade do período de validação, afetando a qualidade dos modelos VAR e VAR-VEC. Os modelos de Box-Jenkins, mais simples e robustos, apresentaram melhores resultados.

Cabe salientar que o modelo VAR para o setor residencial apresentou uma melhor previsão no curto prazo, apenas um horizonte a frente, sendo suplantado pelo modelo ARIMA a partir do segundo horizonte. Já no setor comercial, o melhor MAPE também foi visualizado no modelo VAR, no entanto, somente foi ultrapassado pelo modelo ARIMA no último horizonte de estudo. No setor industrial, o modelo VAR-VEC se mostrou melhor um horizonte a frente, sendo passado pelo modelo ARIMA no segundo horizonte. Desta maneira, chama atenção que, nos três setores consumidores, para a previsão de apenas um passo à frente, os modelos ARIMA apresentaram capacidade preditiva inferior aos modelos obtidos pela outra metodologia, o que de certa forma concorda com os achados de Castro et al. (2016), que fizeram previsões para um horizonte de seis meses.

\section{Referências}

Agência Nacional de Energia Elétrica (2008). Atlas de Energia Elétrica do Brasil. Brasília, DF: ANEEL. Recuperado em janeiro, 2018, de http://www2.aneel.gov.br/arquivos/PDF/atlas3ed.pdf

Akaike, H. (1973). Maximum likelihood identification of Gaussian autoregressive moving average models, Biometrika, 60(2), 255-265. Retrieved January, 2016, from: http://www.jstor.org/stable/2334537?seq=1\#page_scan_tab_contents 
Amaro, R. S., Ceretta, P. S., Coronel, D. A., Bender, R., Filho, Oliveira, M. G. (2017). Comparação da capacidade preditiva de modelos ARIMA para o consumo de energia elétrica no Brasil, Revista Unemat de Contabilidade, 6(11), 173-192. DOI: https://doi.org/10.30681/ruc.v6i11.1848

Andrade, T. A., Lobão, W. J. A. (1997). Elasticidade renda e preço da demanda residencial de energia elétrica no Brasil [Texto para discussão no 489]. Brasília, DF: IPEA. Recuperado em janeiro, 2016, de: http://repositorio.ipea.gov.br/bitstream/11058/2162/1/td_0489.pdf

Box, G. E. P., Jenkins, G. M. (1976). Time series analysis: forecasting and control. 1st ed. New Jersey: Prentice Hall.

Box, G. E.; Jenkins, G. M.; Reinsel, G. C. (1994). Time series analysis: Forecasting and control. 3a ed. New Jersey: Printice Hall.

Bueno, R. D. L. S. (2011). Econometria de séries temporais, 2a ed. São Paulo: Editora Cengage Learning.

Cabral, J. A., Legey, L. F. L., Cabral, M. V. F. (2017). Electricity consumption forecasting in Brazil: A spatial econometrics approach. Energy. V. 126, P. 124-131. DOI: https://doi.org/10.1016/j.energy.2017.03.005

Castro N. R., Gilio, I., Silva, A. F., Ozaki, V. G. (2016). Modelos univariados e multivariados aplicados à previsão de valores de exportação: uma análise comparativa para o complexo soja, Revista Espacios. 37(3). Recuperado em 10 abril, 2020, de: https://www.revistaespacios.com/a16v37n03/16370315.html

Cottrell, A., Lucchetti, R. J. (2018). GRETL: GNU Regression, Econometrics and Timeseries Library. Version 2018d. Recuperado em abril, 2018, de: http://gretl.sourceforge.net/

Dickey, D. A., Fuller, W. A. (1981). Likelihood ratio statistics for autoregressive time series with a unit root [versão eletrônica]. Econometrica: Journal of the Econometric Society, 49(4), 1057-1072. Recuperado em Janeiro, 2018, de: http://www.jstor.org/stable/1912517?seq=1\#page_scan_tab_contents

ELETROBRAS. (2018). Boletim SIESE: consumo de energia elétrica no Brasil. Recuperado em janeiro, 2018, de: http://www.ipeadata.gov.br

Engle, R. (1982). Autoregressive conditional heteroscedasticity with estimates of the variance of United Kingdom inflation. Econometrica, 50(4), 987-1.007. Recuperado em março, 2018, de: https://pdfs.semanticscholar.org/2ee6/cb87fc81ecd78d161c4a92c9dfce00c8961c.pdf?_ga=2. 146146356.1600934247.1590176948-885226569.1590176948

Ferreira, R. V.; Braga, A. P.; Mendes, E. M. A. M. (2006). Previsão de demanda: um estudo de caso para o sistema interligado nacional. Dissertação de Mestrado, Universidade Federal de Minas Gerais, Belo Horizonte, MG, Brasil. Recuperado em agosto, 2019, de: https://www.ppgee.ufmg.br/defesas/417M.PDF

Ferreira, J. S. P.; Silva, N. R. (2015). Determinação dos parâmetros $\alpha$ e b da função de Cobb-Douglas, através do método dos mínimos quadrados. Proceeding Series of the Brazilian Society of Computational and Applied Mathematics, 3(1), 1-2.

DOI: https://doi.org/10.5540/03.2015.003.01.0145 
González-Romera, E., Jaramillo-Morán, M. A., Carmona-Fernández D. (2008). Monthly electric energy demand forecasting with neural networks and Fourier series, Energy Conversion and Management, 49, 3135-3142. DOI: https://doi.org/10.1016/j.enconman.2008.06.004

Gujarati, D. N., Porter, D. C. (2011). Econometria Básica. 5a ed. São Paulo, SP: AMGH Editora.

Hannan, E. J., Quinn, B. G. (1979). The determination of the order of an autoregression, Journal of the Royal Statistical Society - Series B, 41, 190-195.

Irffi, G., Castelar, I., Siqueira, M. L., Linhares, F. C. (2009). Previsão da demanda por energia elétrica para classes de consumo na região Nordeste, usando OLS dinâmico e mudança de regime [versão eletrônica], Economia Aplicada, 13(1), 69-98. Recuperado em janeiro, 2018, de: http://www.scielo.br/pdf/ecoa/v13n1/v13n1a04.pdf

Jarque, C., Bera, A., (1987). A test for normality of observations and regression residuals. International Statistical Review 55, 163-172. DOI: https://doi.org/10.2307/1403192

Johansen, S. (1988). Statistical analysis of cointegration vectors [versão eletrônica], Journal of economic dynamics and control, 12(2-3), 231-254. Recuperado em janeiro, 2018, de: http://www.sciencedirect.com/science/article/pii/0165188988900413

Kwiatkowski, D., Philips, P. C. B., Schimdt, P., Schin, Y. (1992). Testing the null hypothesis of stationarity against the alternative of a unit root: How sure are we that economic time series have a unit root [versão eletrônica], Journal of econometrics, 54(1-3), 159-178. Recuperado em janeiro, 2018, de: http://www.sciencedirect.com/science/article/pii/030440769290104Y

Leite, S. Q. (2006). Projeções para a demanda por energia elétrica no Brasil, 2006-2015. Dissertação de Mestrado Profissionalizante, Faculdades Ibemec, Rio de Janeiro, RJ, Brasil. Recuperado em junho, 2018, de: http://s3.amazonaws.com/public-cdn.ibmec.br/portalibmeccontent/public/arquivos/df/dis_2006_12_-_sidimar_quezada_leite.pdf

Lima, H. C. G. (2011). A demanda setorial de energia elétrica em Pernambuco. Dissertação de Mestrado, Universidade Federal de Pernambuco, Recife, PE, Brasil. Recuperado em janeiro, 2019, de: http://repositorio.ufpe.br/handle/123456789/1240

Ljung, G.M., Box, G.E.P. (1978). On a Measure of Lack of Fit in Time Series Models. Biometrika, 65(2), 297-303.

Mattos, L. B. (2004). Demanda de energia elétrica no estado de Minas Gerais: 1970-2002. Dissertação de Mestrado, Universidade Federal de Viçosa, MG, Brasil. Recuperado em novembro, 2018, de: https://q.eletrobras.com/pt/EstudantesePesquisadores/biblioteca/Demanda\%20de\%20Energi a\%20El\%C3\%A9trica\%20no\%20Estado\%20de\%20Minas\%20Gerais_\%201970-2002.PDF

Mattos, L. B., Lima, J. E. de. (2005). Demanda residencial de energia elétrica em Minas Gerais: 19702002 [versão eletrônica]. Nova Economia, 15(3), 31-52. DOI: https://doi.org/10.1590/S010363512005000300002

Modiano, E. M. (1984). Elasticidade-renda e preços da demanda de energia elétrica no Brasil [Texto para discussão, 68]. Rio de Janeiro, RJ: PUC. Recuperado em janeiro, 2019, de: http://www.econ.puc-rio.br/uploads/adm/trabalhos/files/td68.pdf 
Morettin, P. A. (2006). Econometria Financeira: Um curso em Séries Temporais Financeiras. São Paulo: Editora Edgard Blucher.

R Core Team (2019). R: A Language and Environment for Statistical Computing. R Foundation for Statistical Computing. Vienna, Áustria: R Core Team. Recuperado em abril, 2019, de: https://www.R-project.org

Schmidt, C. A. J., Lima, M. A. (2004). A demanda por energia elétrica no Brasil, Revista Brasileira de Economia, 58(1), 67-98. DOI: https://doi.org/10.1590/S0034-71402004000100004

Schwarz, G. (1978). Estimating the dimension of a model [versão eletrônica], Annals of Statistics, 6(2), 461-464. Recuperado em dezembro, 2017, de http://qwone.com/ jason/trg/papers/schwarzdimension-78.pdf

Silveira, A. G. (2017). Estudo da demanda energia elétrica no Brasil. Dissertação de Mestrado, Universidade Federal de Rio Grande (FURG), Rio Grande, RS, Brasil. Recuperado em março, 2018, de: https://ppgmc.furg.br/puclica

Viana, G. I. M. N. (2010). Um modelo para projeções para demanda por energia elétrica, 2009-2017 e a evolução do custo social e tarifa ótima para o Brasil. Dissertação de Mestrado, Universidade Federal de Alagoas, Maceió, AL, Brasil. Recuperado em dezembro, 2018, de: http://www.repositorio.ufal.br/jspui/bitstream/riufal/1377/1/Um\%20modelo\%20para\%20proj e\%c3\%a7\%c3\%b5es\%20para\%20demanda\%20por\%20energia\%20el\%c3\%a9trica\%2c\%202009 $-$ 2017\%20e\%20a\%20evolu\%c3\%a7\%c3\%a3o\%20do\%20custo\%20social\%20e\%20tarifa\%20\%c3 \%b3tima\%20para\%20o\%20Brasil.pdf

Viana, G. I. M. N., Silva, A. L. M. (2014). Um modelo para projeções para demanda por energia elétrica, 2009-2017 para o setor residencial no Brasil [versão eletrônica], Revista Brasileira de Energia, 20(1), 107-126. Recuperado em fevereiro, 2018, de: http://new.sbpe.org.br/artigo/327/ 
Apêndice 1

Fluxograma da metodologia VAR-VEC

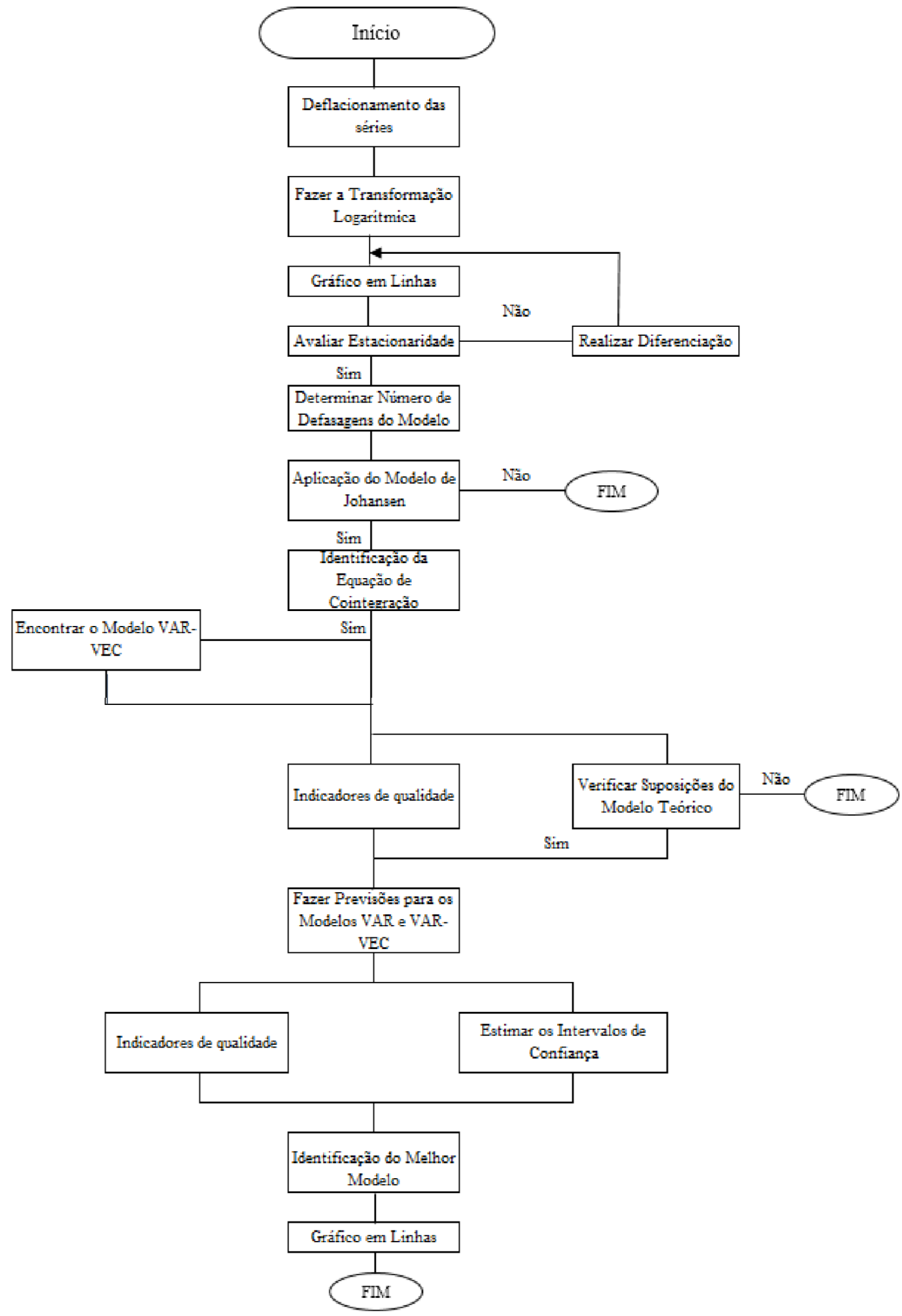

Fonte: Elaborado pelo autor, adaptado de Bueno (2011). 


\section{Apêndice 2}

Fluxograma da metodologia Box-Jenkins

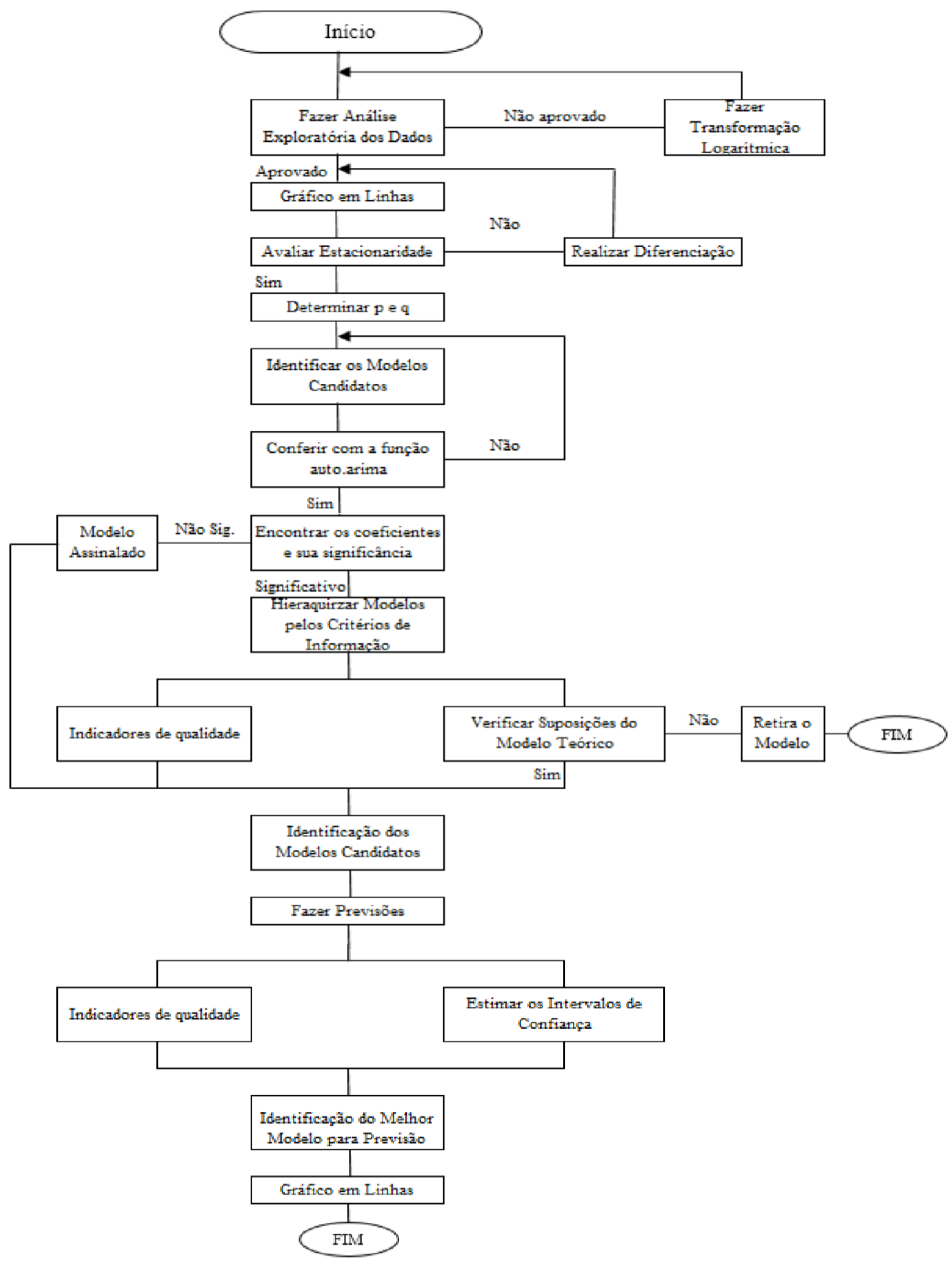

Fonte: Elaborado pelo autor, Adaptado de Box et al (1994) e Gujarati e Porter (2011). 